\title{
Cluster observations of sudden impulses in the magnetotail caused by interplanetary shocks and pressure increases
}

\author{
K. E. J. Huttunen ${ }^{1}$, J. Slavin ${ }^{2}$, M. Collier ${ }^{2}$, H. E. J. Koskinen ${ }^{1,3}$, A. Szabo ${ }^{2}$, E. Tanskanen ${ }^{2}$, A. Balogh ${ }^{4}$, E. Lucek ${ }^{4}$, and \\ H. Rème ${ }^{5}$ \\ ${ }^{1}$ Department of Physical Sciences, P.O. Box 64, FIN-00014 University of Helsinki, Finland \\ ${ }^{2}$ Laboratory for Extraterrestial Physics, NASA Goddard Space Flight Center, Greenbelt, Maryland \\ ${ }^{3}$ Finnish Meteorological Institute, P.O. Box 503, 00101 Helsinki, Finland \\ ${ }^{4}$ Blackett Laboratory, Imperial College, London, England \\ ${ }^{5}$ Centre d'Etude Spatiale des Rayonnements, Toulouse, France
}

Received: 17 August 2004 - Revised: 18 November 2004 - Accepted: 8 December 2004 - Published: 28 February 2005

\begin{abstract}
Sudden impulses (SI) in the tail lobe magnetic field associated with solar wind pressure enhancements are investigated using measurements from Cluster. The magnetic field components during the SIs change in a manner consistent with the assumption that an antisunward moving lateral pressure enhancement compresses the magnetotail axisymmetrically. We found that the maximum variance SI unit vectors were nearly aligned with the associated interplanetary shock normals. For two of the tail lobe SI events during which Cluster was located close to the tail boundary, Cluster observed the inward moving magnetopause. During both events, the spacecraft location changed from the lobe to the magnetospheric boundary layer. During the event on 6 November 2001 the magnetopause was compressed past Cluster. We applied the 2-D Cartesian model developed by Collier et al. (1998) in which a vacuum uniform tail lobe magnetic field is compressed by a step-like pressure increase. The model underestimates the compression of the magnetic field, but it fits the magnetic field maximum variance component well. For events for which we could determine the shock normal orientation, the differences between the observed and calculated shock propagation times from the location of WIND/Geotail to the location of Cluster were small. The propagation speeds of the SIs between the Cluster spacecraft were comparable to the solar wind speed. Our results suggest that the observed tail lobe SIs are due to lateral increases in solar wind dynamic pressure outside the magnetotail boundary.
\end{abstract}

Key words. Interplanetary physics (Interplanetary shocks) - Magnetospheric physics (Magnetotail; Solar windmagnetosphere interaction)

\section{Introduction}

The impact of an interplanetary shock causes global changes in the magnetosphere. Solar wind pressure increases across the shock, pushing the magnetopause inward. As a consequence, there are changes in magnetospheric and ionospheric current systems (e.g. Chapman-Ferraro, Region 1 and crosstail currents). An impulsive compression of the subsolar magnetopause launches a hydromagnetic wave that propagates in about one minute to the ionosphere. Low-latitude magnetograms record rapid perturbations called sudden impulses (SI), or storm sudden commencements (SSC) if a geomagnetic storm follows, see (Araki, 1977; Smith et al., 1986; Araki, 1994; Lee and Hudson, 2001). Especially for the southward interplanetary magnetic field (IMF), pressure pulses cause almost immediate and global enhancements in ionospheric currents and auroral precipitation, see (Zhou and Tsurutani, 1999; Zesta et al., 2000; Boudouridis et al., 2003; Meurant et al., 2003). In the tail lobes, sudden increases in the magnetic field have been observed on time scales from a few to ten minutes, see (Sugiura et al., 1968; Kawano et al., 1992; Collier et al., 1998; Kim et al., 2004).

Two mechanisms have been suggested as the cause of the tail lobe SIs: 1) The transfer of magnetic flux from the dayside to the tail by magnetohydrodynamic (MHD) waves resulting from the sudden enhancement of solar wind pressure on the front of the magnetosphere (Sugiura et al., 1968); 2) Compression of the magnetotail by magnetosheath pressure increases, see (Kawano et al., 1992; Collier et al., 1998). In the first scenario the disturbance propagates at the Alfvén speed. The latter mechanism assumes that the magnetotail remains in equilibrium as a solar wind pressure enhancement moves antisunward, compressing its radius axisymmetrically (Fig. 1).

Correspondence to: E. Huttunen

(emilia.huttunen@helsinki.fi) 


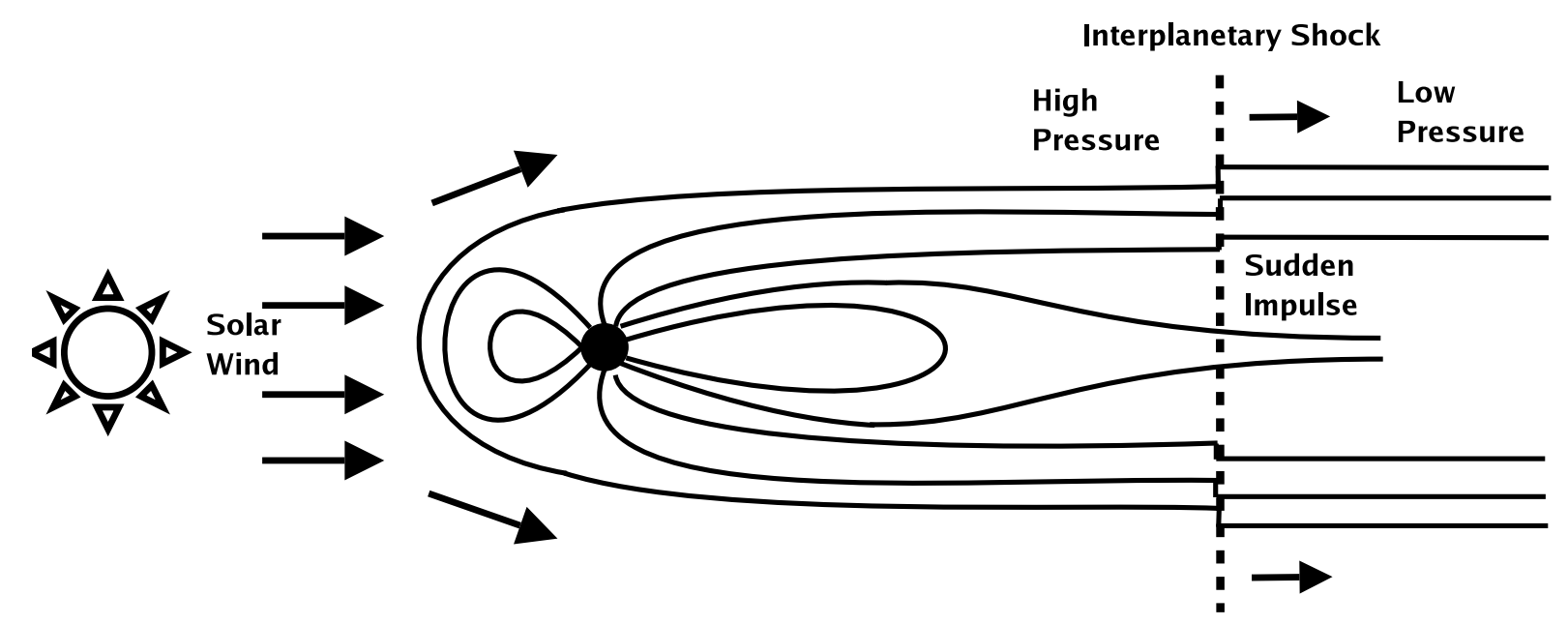

Fig. 1. A sketch showing the deformation of the magnetotail by an interplanetary shock that moves antisunward causing a sudden increase in the tail lobe magnetic field.

The pressure balance equation between the tail lobe and solar wind pressures can be expressed as (Slavin et al., 1983):

$\frac{B_{\text {lobe }}^{2}}{2 \mu_{0}}=K P_{\text {dyn }}^{S W} \sin ^{2} \alpha+\frac{B_{S W}^{2}}{2 \mu_{0}}+n_{S W} k_{b} T_{S W}$

where $T_{S W}$ is the sum of the solar wind electron and proton temperatures, and $P_{\text {dyn }}^{S W}\left(=n_{S W} m v_{S W}^{2}\right), B_{S W}, n_{S W}$ are solar wind dynamic pressure, magnetic field and ion density. The plasma pressure in the lobe has been assumed negligible. The parameter $K$ is a "drag coefficient", a measure of how efficiently solar wind particles transfer momentum to the magnetopause. Spreiter et al. (1966) determined that a value of 0.881 describes the pressure exerted by a hypersonic flow around the magnetopause. In Eq. (1) above, $\alpha$ is the flaring angle, i.e. the angle between the solar wind flow and the tail boundary. At the distances $10-20 R_{E}$ downtail from the Earth, the flaring angle varies from $17^{\circ}$ to $30^{\circ}$, depending on the solar wind dynamic pressure (Nakai et al., 1991). Since the Cluster events we considered were located in the nearEarth tail $\left(\sim 10-19 R_{E}\right)$, the tail flaring was significant and the tail compression was caused mainly by increased $P_{\text {dyn }}^{S W}$. What is actually relevant for magnetopause compression is the pressure profile in the magnetosheath. However, as discussed in Collier et al. (1998), the pressure discontinuities in the solar wind are transmitted through the bow shock to form similar structures in the magnetosheath.

The previous studies, except Kim et al. (2004), have used data from only a single spacecraft. The multi-spacecraft Cluster mission allows us to calculate the propagation speed of the disturbance between the four Cluster spacecraft and also, if crossed, the propagation speed of the magnetopause. Furthermore, Cluster plasma data provide details of the tail compression, e.g. the motion of the magnetopause towards Cluster. The two tail lobe SIs studied in detail by Collier et al. (1998) were relatively weak, with the magnetic field increases 4.2 and $3 \mathrm{nT}$ (from 15 and $10 \mathrm{nT}$, respectively) in response to small increases in the solar wind dynamic pressure. The SI events investigated in this study are associated with strong interplanetary shocks or substantial enhancements in the solar wind dynamic pressure and consequently much larger increases in the lobe magnetic field magnitude. Upstream solar wind coverage by ACE, WIND, and Geotail allowed us to calculate the shock normal orientation and the shock speed and to perform reliable timing analyses. In Sect. 2 we describe the properties of the interplanetary shocks and the associated tail lobe SI events. In Sect. 3 we present the Collier et al. (1998) model and the results of the least-squares fittings of this model to the magnetic field data. In Sect. 4 we present the results of the timing analysis and in Sect. 5 we discuss events during which the Cluster location changed from the lobe to the magnetospheric boundary layer/magnetosheath. Finally, in Sect. 6 we summarize and discuss the significance of our results.

\section{Event description}

\subsection{Event selection}

Cluster is composed of four spacecraft: Rumba (C1), Salsa (C2), Samba (C3) and Tango (C4). The first pair of Cluster satellites (Salsa and Samba) were launched on 16 July 2000 and the second pair (Rumba and Tango) on 9 August 2000. We have investigated two time periods covering JulyNovember 2001 and July-November 2002. These time intervals were selected because the Cluster apogee was beyond $-10 R_{E}$. We also checked the data for the July-November 2003, but did not find any interplanetary shocks while Cluster was in the tail lobe with data available. Furthermore, during 2003 the separation of Cluster satellites was too small $(\sim 500 \mathrm{~km})$ to take full advantage of the multi-spacecraft observations. In late 2001 the separation between the spacecraft at the apogee was $\sim 0.1-0.3 R_{E}$ and increased to $\sim 1-$ $3 R_{E}$ late in 2002. In this study we have used data from the 


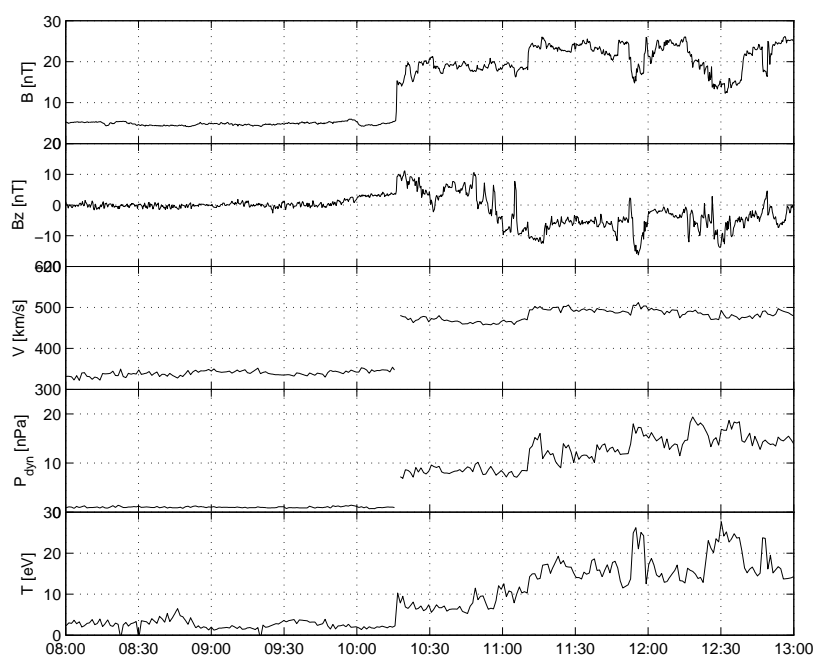

Fig. 2. ACE magnetic field and plasma measurements for an interplanetary shock observed on 17 August 2001 at 10:16 UT. Panels show magnetic field intensity, the IMF $Z$ component in GSM coordinate system, plasma speed, dynamic pressure and proton temperature. The shock was associated with a tail lobe SI observed by Cluster at 11:07 UT at a distance $X=-18.3 R_{E}$ downtail.

FGM (Fluxgate Magnetometer), PEACE (Plasma Electron And Current Experiment) and CIS (Cluster Ion Spectrometer) instruments. For the magnetic field we used both 4-s and high resolution data.

We used data from ACE, WIND and Geotail to identify and investigate shocks in the solar wind, together with the ACE interplanetary shock list at: http://www.bartol.udel.edu/ $\sim$ chuck/ace/ACElists/obs_list.html+. The investigated period occured during solar maximum, so interplanetary shocks were frequently observed near $1 \mathrm{AU}$. For each shock we checked whether Cluster was located in the tail lobe or in the plasma sheet boundary layer (PSBL) $\sim 10 R_{E}$ or further down the tail. The tail lobes are characterized by high and steady magnetic field strength $(\sim 20 \mathrm{nT})$, large negative (south lobe) or positive (north lobe) magnetic field $X$ components, lower ion densities and temperatures than observed in the magnetosheath or in the plasma sheet, and low plasma betas (less than 0.1). All interplanetary shocks which occurred while Cluster was positioned as described above were related to tail lobe SIs. In total, we found 10 events.

\subsection{Interplanetary shocks}

The interplanetary shocks associated with the tail lobe SIs are listed in Table 1. The time of the shock is given as observed at ACE and determined from the 16-s resolution magnetic field data. For each shock the shock normal orientation, $\mathbf{n}_{\mathbf{s h}}$, was estimated using the nonlinear least squares fitting technique of the Rankine-Hugoniot jump conditions by Szabo (1994). A more thorough analysis of these shocks revealed that the 29 September 2001 and 26 August 2002 cases did not represent valid shocks. However, we included these

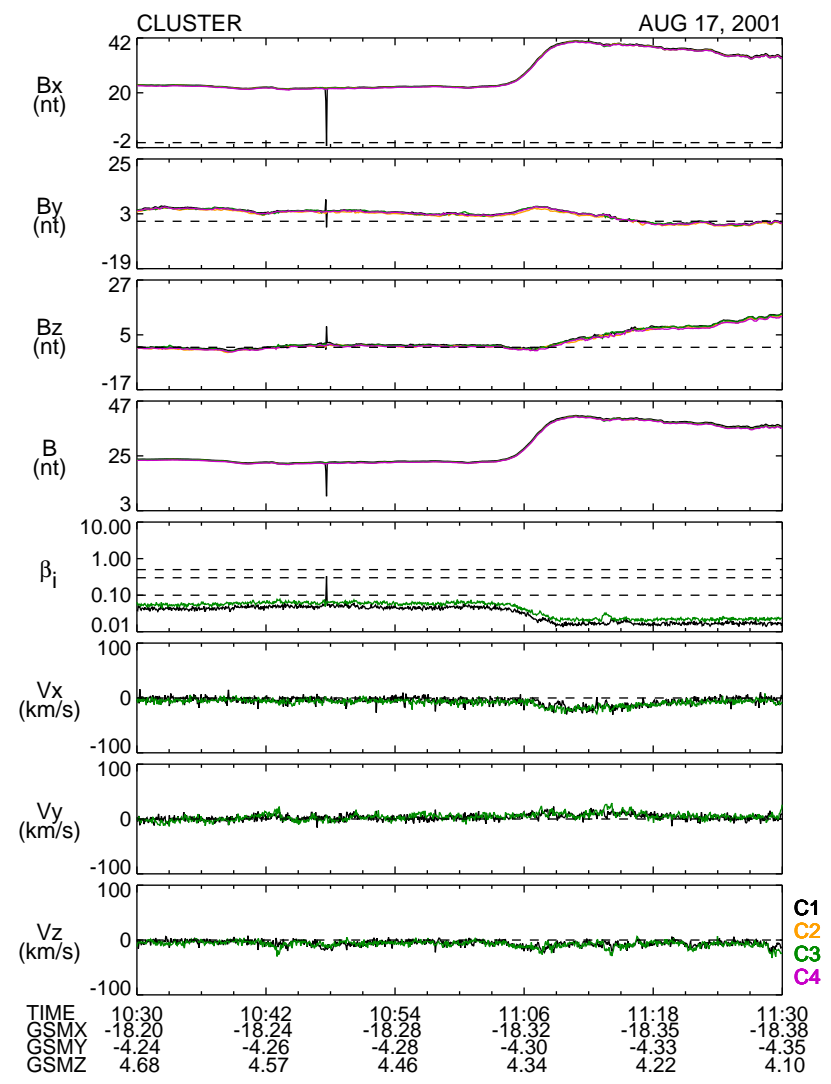

Fig. 3. Cluster FGM and CIS (from C1 and C3) observations for the tail lobe SI event observed on 17 August 2001 over a 1-h interval. Panels show magnetic field components in the GSM coordinate system, the magnetic field intensity, plasma beta, and the components of the velocity.

two events in the study because they were associated with substantial and rapid solar wind pressure increases and tail lobe SIs. The variations in solar wind parameters across the shocks were calculated using 20-min averaged data upstream (u) and downstream (d) of the shock.

Figure 2 shows a shock that was observed on 17 August 2001 at 10:16 UT by ACE. The shock is identified by clear and sudden jumps in the magnetic field magnitude, solar wind speed, dynamic pressure and proton temperature. Table 1 shows that the shocks investigated in this study were strong. The shocks on 25 October 2001 and on 18 August 2002 had Alfvénic Mach numbers of 6.7 and 8.4, respectively. For the rest of the shocks, the Mach numbers ranged from 2.4 to 3.5. The typical Mach number for the solar maximum period is between 2-4, but in extreme cases values as high as 9 are observed (Echer et al., 2003). The change in the solar wind speed across the shock exceeded $100 \mathrm{~km} / \mathrm{s}^{-1}$ for all of the other events except for 25 July 2002. The increases in the magnetic field magnitude and dynamic pressure were also substantial. 
Table 1. Shocks and pressure increases (events 2,9) associated with the tail lobe SI events. Columns from left to right give the event number $(\mathrm{N})$, time of the shock, shock normal $\left(\mathbf{n}_{\mathbf{s h}}\right)$ in GSM, shock speed in the $X$-direction $\left(V_{S X}\right)$, changes in the magnetic field magnitude, solar wind speed and dynamic pressure across the shock, and upstream (u) and downstream (d) magnetic field north-south components. The last two columns give the Alfvénic Mach number $\left(M_{a}\right)$, and the angle between the upstream magnetic field and the shock normal $\left(\theta_{\mathrm{Bun}}\right)$.

\begin{tabular}{|c|c|c|c|c|c|c|c|c|c|c|}
\hline $\mathrm{N}$ & time (UT) & $\begin{array}{l}\hat{\mathbf{n}} \\
\left(\mathrm{km} / \mathrm{s}^{-1}\right)\end{array}$ & $\begin{array}{l}V_{S X} \\
\text { (nT) }\end{array}$ & $\begin{array}{r}\Delta B \\
\left(\mathrm{~km} / \mathrm{s}^{-1}\right)\end{array}$ & $\begin{array}{r}\Delta V \\
(\mathrm{nPa})\end{array}$ & $\begin{array}{r}\Delta P_{\text {dyn }} \\
\quad(\mathrm{nT})\end{array}$ & $\begin{array}{l}B_{Z u} \\
(\mathrm{nT})\end{array}$ & $B_{Z d}$ & $\begin{array}{r}M_{a} \\
\left(^{\circ}\right)\end{array}$ & $\theta_{\text {Bun }}$ \\
\hline & 2001 & & & & & & & & & \\
\hline 1 & $17.810: 16$ & $(0.99,-0.16,0.03)$ & 522 & 14.1 & 125 & 7.3 & 2.5 & 4.4 & 3.5 & 87.1 \\
\hline 2 & 29.9 09:06 & not a shock & 633 & 3.9 & 120 & 4.2 & 0.2 & -2.7 & - & - \\
\hline 3 & $11.1016: 20$ & $(0.98,0.12,0.19)$ & 531 & 10.4 & 138 & 13.9 & 1.2 & -13.5 & 2.5 & 65.7 \\
\hline 4 & $25.1008: 02$ & $(0.89,0.28,-0.37)$ & 414 & 5.0 & 107 & 7.2 & 0.5 & 4.3 & 6.14 & 42.2 \\
\hline 5 & $28.1002: 42$ & $(0.93,-0.37,0.01)$ & 529 & 12.7 & 143 & 4.4 & -4.4 & -15.4 & 2.8 & 42.7 \\
\hline 6 & $\begin{array}{l}6.1101: 20 \\
2002\end{array}$ & no plasma data & - & 60 & & & & & & \\
\hline 7 & $25.712: 59$ & $(0.82,0.35,-0.44)$ & 506 & 4.9 & 55 & 1.0 & 5.7 & 8.5 & 2.4 & 85.3 \\
\hline 8 & $18.818: 10$ & $(0.97,-0.17,-0.20)$ & 602 & 5.0 & 158 & 9.1 & 0.7 & 1.4 & 8.4 & 57.9 \\
\hline 9 & $26.810: 45$ & not a shock & 383 & 5.6 & 79 & 4.7 & -3.8 & 1.3 & - & - \\
\hline 10 & $7.916: 09$ & $(0.86,0.49,0.14)$ & 524 & 15.9 & 180 & 7.6 & -7.6 & -21.9 & 3.0 & 76.5 \\
\hline
\end{tabular}

Table 2. Tail lobe SI events. Values are taken from C3, except for 18 August 2002, for which the C1 data were used. Panels show: the initial magnetic field magnitude $\left(B_{0}\right)$, the total amount of the change in $B_{x}$ (the percentage of the $B_{0}$ shown in parenthesis), the amplitude of the change in GSM $B_{y}$ and $B_{z}$, the start time $\left(T_{S}\right)$ and the duration of the SI, the direction of the maximum variance vector $\hat{\mathbf{e}}_{\mathbf{x}}^{*}$ in GSM, the eigenvalue ratio $\left(\lambda_{y}^{*} / \lambda_{z}^{*}\right)$, and the C3 location in GSM.

\begin{tabular}{llrrllrlrrrr}
\hline $\mathrm{N}$ & $\begin{array}{l}B_{0} \\
(\mathrm{nT})\end{array}$ & $\begin{array}{r}\Delta B_{x} \\
(\mathrm{nT})\end{array}$ & $\begin{array}{r}\Delta B_{y} \\
(\mathrm{nT})\end{array}$ & $\begin{array}{l}\Delta B_{z} \\
(\mathrm{nT})\end{array}$ & $\begin{array}{l}T_{S} \\
(\mathrm{UT})\end{array}$ & $\begin{array}{r}\mathrm{T} \\
(\mathrm{min})\end{array}$ & $\hat{\mathbf{e}}_{\mathbf{x}}{ }^{*}$ & $\lambda_{y}^{*} / \lambda_{z}^{*}$ & $\begin{array}{r}X_{C} \\
\left(R_{E}\right)\end{array}$ & $\begin{array}{r}Y_{C} \\
\left(R_{E}\right)\end{array}$ & $\begin{array}{r}Z_{C} \\
\left(R_{E}\right)\end{array}$ \\
\hline 1 & 22.5 & $+18.0(80 \%)$ & +4.5 & - & $11: 03: 30$ & 7.33 & $(0.99,0.00,0.13)$ & 25.5 & -18.32 & -4.29 & 4.34 \\
2 & 26.0 & $-11.5(44 \%)$ & +5.0 & - & $09: 39: 10$ & 6.00 & $(0.95,0.16,-0.28)$ & 1.6 & -17.37 & 6.95 & -4.75 \\
3 & 38.0 & $-27.0(71 \%)$ & +16.0 & -7.0 & $17: 01: 40$ & 3.17 & $(0.94,0.25,0.22)$ & 5.1 & -11.81 & 9.12 & -9.33 \\
4 & 15.0 & $-13.5(90 \%)$ & +9.0 & -3.5 & $08: 50: 30$ & 2.83 & $(0.82,0.45,0.36)$ & 35.9 & -12.35 & 13.40 & -4.76 \\
5 & 31.5 & $-24.5(79 \%)$ & +12.5 & -3.5 & $03: 19: 30$ & 6.83 & $(0.95,-0.30,0.06)$ & 14.7 & -10.20 & 14.43 & -7.00 \\
6 & 20.0 & & & & & & & & -9.45 & 14.93 & 2.37 \\
7 & 34.0 & $+5.5(16 \%)$ & +3.0 & -2.5 & $13: 37: 50$ & 7.83 & $(0.85,0.46,-0.28)$ & 19.0 & -9.67 & -7.92 & 9.48 \\
8 & 22.0 & $-20(90 \%)$ & +10 & - & $18: 47: 20$ & 6.00 & $(0.96,-0.21,-0.21)$ & 1.7 & -18.18 & -5.17 & 1.58 \\
9 & 34.5 & $-15(43 \%)$ & -8.0 & -2.0 & $11: 30: 30$ & 10.67 & $(0.89,0.45,0.03)$ & 3.9 & -16.40 & -3.46 & -6.09 \\
10 & 54.0 & $-24(44 \%)$ & +3.0 & -8.0 & $16: 36: 50$ & 5.17 & $(0.96,0.26,0.14)$ & 19.0 & -9.95 & 0.23 & -10.26 \\
\hline
\end{tabular}

\subsection{Tail lobe SI events}

Table 2 lists the tail lobe SIs associated with the interplanetary shocks in Table 1 . The Cluster measurements during the tail lobe SI corresponding to the shock in Fig. 2 are presented in Fig. 3 for a 1-h period. At the time of the SI Cluster was located in the northern tail lobe at the GSM-position $(X, Y$, $Z)=(-18.32,-4.29,4.33) R_{E}$ approaching the plasma sheet. The tail lobe is identified from the steady and large GSM magnetic field $X$ component and small plasma beta. The plasma beta decreases as the magnetic field magnitude increases during the compression. Figure 4 presents magnetic field data for the 12-min interval around the same SI event. $B_{x}$ increased smoothly from $22.5 \mathrm{nT}$ to about $40.5 \mathrm{nT}$. The rise time between the asymptotic values was about $7 \mathrm{~min}$ and the most rapid increase occurred in 3-4 min. $B_{y}$ exhibited a small, positive deflection with the amplitude of $+4.5 \mathrm{nT}$ as $B_{x}$ made its transition. The $B_{z}$ component increased slowly from 2 to $4 \mathrm{nT}$. Figure 5a shows a schematic description of the expected changes in the magnetic field components during a step-like compression in the north lobe in the XY plane. The $B_{x}$ increase is illustrated by the converging field lines. When the spacecraft is located in the northern dawn lobe (negative $Y$-coordinate) the direction of the $B_{y}$ deflection is positive and when located in the northern dusk lobe (positive $Y$-coordinate) the deflection is negative.

Similarly, for other SI events the increase in the magnetic field magnitude was primarily due to the increase in $B_{x}$ (north lobe) or due to the decrease in the initially negative $B_{x}$ (south lobe). All SIs, except the one on 25 July 2002, which was associated with the weakest shock in this study, had an 


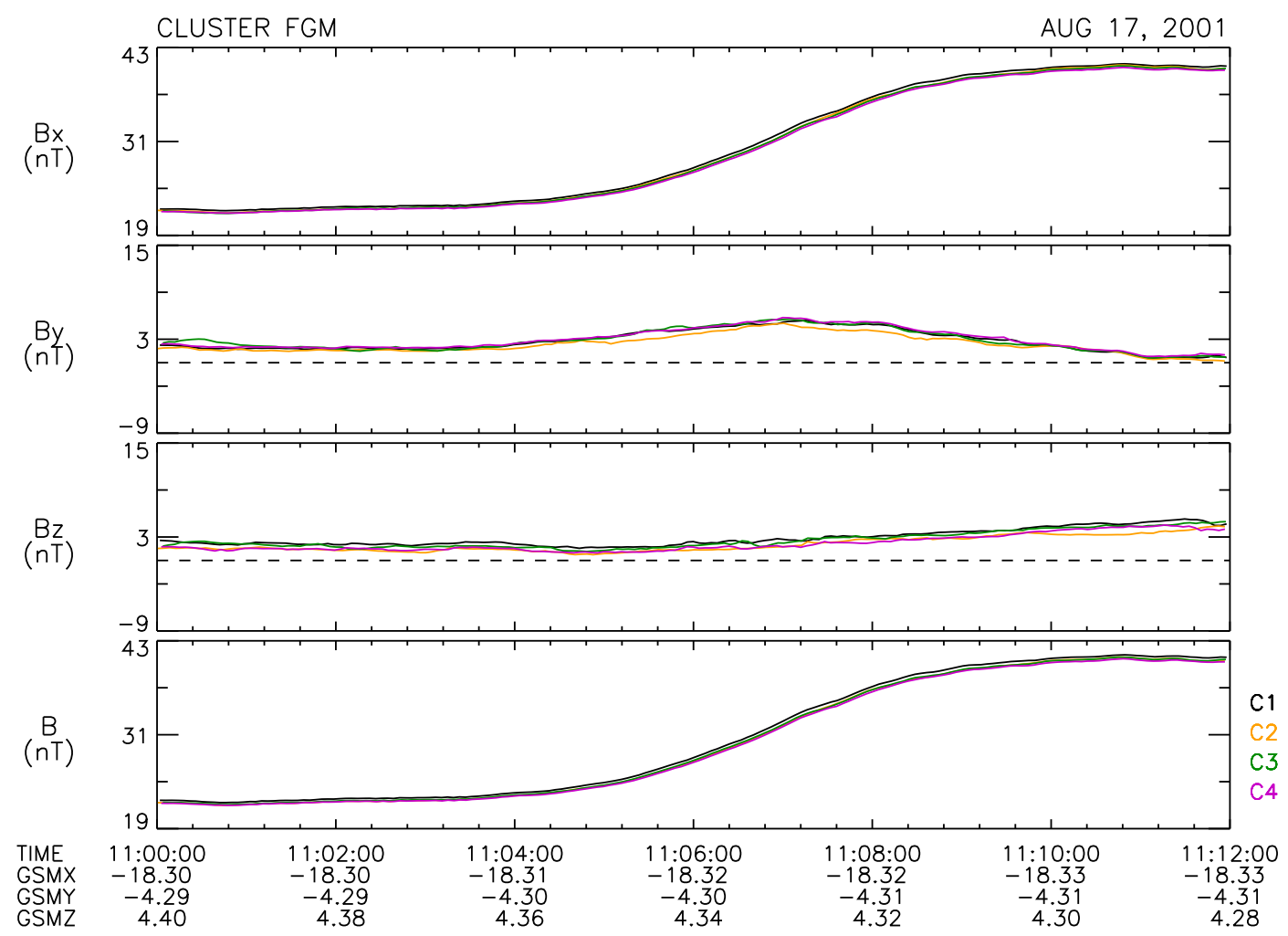

Fig. 4. Fine scale plot of the tail lobe SI presented in Fig. 3. Panels show the magnetic field components in the GSM coordinate system and the magnetic field magnitude.

increase larger than $10 \mathrm{nT}$ in the magnetic field magnitude (about $45 \%$ or more of the initial value). The durations of the SI events ranged between $\sim 3-10 \mathrm{~min}$, although most of the magnetic field increase occurred in a shorter time interval. For the 6 November 2001 event the largest change occurred in $B_{z}$. Before the compression Cluster was located close to the magnetotail boundary near the plasma sheet. The magnetopause was compressed beyond Cluster during this event.

In order to examine the magnetic field rotation in more detail we applied the minimum variance analysis (MVA) (Sonnerup and Cahill, 1967) to the Cluster magnetic field data for each SI event. $B_{x}^{*}, B_{y}^{*}$ and $B_{z}^{*}$ denote the magnetic field components in the directions of maximum, intermediate and minimum variance. Figure 6 shows the hodograms of the SI magnetic field vector in the plane of maximum variance and in the plane of minimum variance for the 17 August 2001 event. The maximum variance eigenvector, $\hat{\mathbf{e}}_{\mathbf{x}}^{*}=(0.99,0.01$, 0.13 ), was almost exactly aligned with the GSM $X$-axis and the intermediate variance eigenvector $\hat{\mathbf{e}}_{\mathbf{y}}^{*}=(0.02,0.97,-0.24)$ almost parallel to the GSM $Y$-direction. The large ratio of the intermediate eigenvalue $\left(\lambda_{y}^{*}\right)$ to the minimum eigenvalue $\left(\lambda_{z}^{*}\right)$ indicates that the eigenvectors are well-defined. The statistical study of 43 tail lobe SIs by Kawano et al. (1992) demonstrated that the field rotation is mainly in the $\hat{\mathbf{e}}_{\mathbf{x}}^{*} \hat{\mathbf{e}}_{\mathbf{y}}^{*}$ plane, consistent with the assumption that tail lobe SIs are caused by the constriction in the tail radius. The hodograms show that the projection of the magnetic field into the plane of maximum variance rotates smoothly. The rotation is confined to one plane, as $B_{z}^{*}$ stays almost constant. For all nine SI events investigated using MVA (excluding the 6 November 2001 event) the similar smooth rotation of the magnetic field was observed in one plane. Note that when the magnetotail is not deflected during the compression, the initial and final directions of the magnetic field vector are the same (Fig. 5). The eigenvectors are all well-defined except for the 29 September 2001 and 18 August 2001 SI events. During these two events, Cluster was located near the plasma sheet which caused irregular magnetic field behavior.

The left panel of Fig. 7 shows that the obtained SI maximum variance vectors were roughly parallel with the $X$ axis and almost parallel with the associated shock normal directions when projected on the GSM XZ-plane. Perhaps the post-shock solar wind flow has deflected the magnetotail parallel to itself. The right panel of Fig. 7 shows that the intermediate unit vectors are aligned with the GSM $Y$-axis near the XY-plane, and the vectors turn more aligned with the GSM $Z$-axis when the distance from the XY plane increases.

\subsection{Correlation between shock properties and tail lobe SIs}

Figure 8a shows the comparison between the changes in the solar wind dynamic pressure at $\operatorname{ACE}\left(\Delta P_{\text {dyn }}^{S W}\right)$ and the amplitude of the tail lobe SI event at Cluster $\left(\Delta B_{\text {lobe }}\right)$. The linear correlation coefficient is 0.73 which indicates that the large 


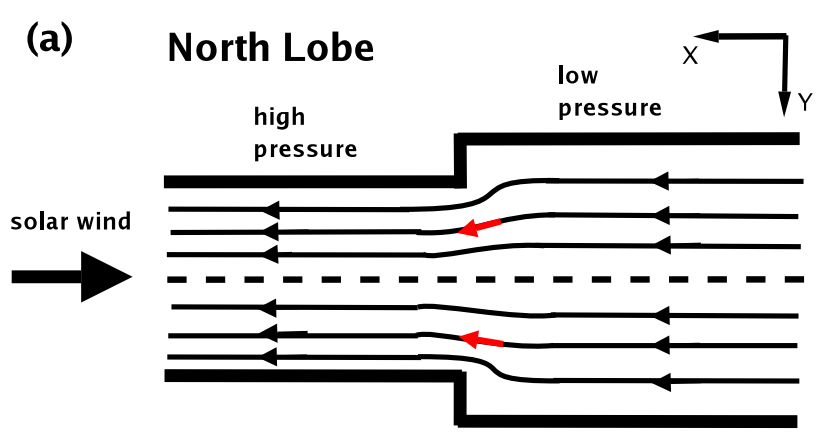

(b)

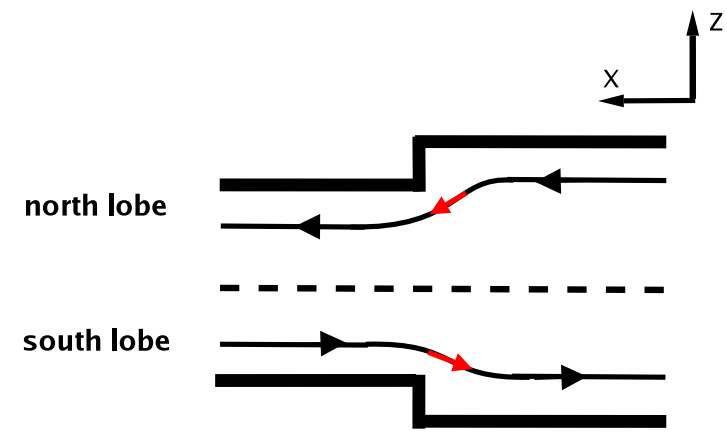

Fig. 5. Sketch of the step-like compression in the $X Y$ and $X Z$ planes. Thin lines represent the magnetic field lines and the thick solid lines represent the tail boundary. Panel (a) shows compression in the north lobe XY plane and panel (b) shows compression in the $\mathrm{XZ}$ plane.

changes in solar wind dynamic pressure cause the largest tail lobe SI events.

The theoretical relationship between $\Delta B_{\text {lobe }}$ and $\Delta P_{\text {dyn }}^{S W}$ can be calculated from Eq. (1):

$$
\Delta B_{\text {lobe }}=\sqrt{2 \mu_{0} K} \times\left(\sqrt{P_{\mathrm{dyn} 2}^{S W}} \sin \alpha_{2}-\sqrt{P_{\mathrm{dyn} 1}^{S W}} \sin \alpha_{1}\right) .
$$

If the subsolar magnetopause distance and the asymptotic tail radius change by the same fraction during the compression then the tail flaring remains constant. The subsolar standoff distance varies as $\left(P_{\mathrm{dyn}}^{S W}\right)^{-1 / 6}$ and the asymptotic tail radius as the $\left(P_{S}^{S W}\right)^{-1 / 4}$, where $P_{S}^{S W}$ is the solar wind static pressure $\left(2 n_{S W} k_{b} T_{S W}+B_{S W}^{2} / 2 \mu_{0}\right)$. Based on these dependencies Collier et al. (1998) introduced a parameter $S$ as the measure of the degree of self-similarity in the magnetopause shape:

$S=\frac{\left(P_{\mathrm{dyn}}^{S W}\right)^{2}}{\left(P_{S}^{S W}\right)^{3}}$.

If the tail radius is compressed more than the subsolar point, then the tail flaring decreases. When the subsolar point is compressed more than the tail radius, then the tail flaring increases. The dashed line in Fig. 8a shows the relationship from Eq. (2) using a constant value of $17^{\circ}$ for $\alpha$ which predicts too large increase in the tail lobe magnetic field. We calculated the values of $S$ for each event across the shock using
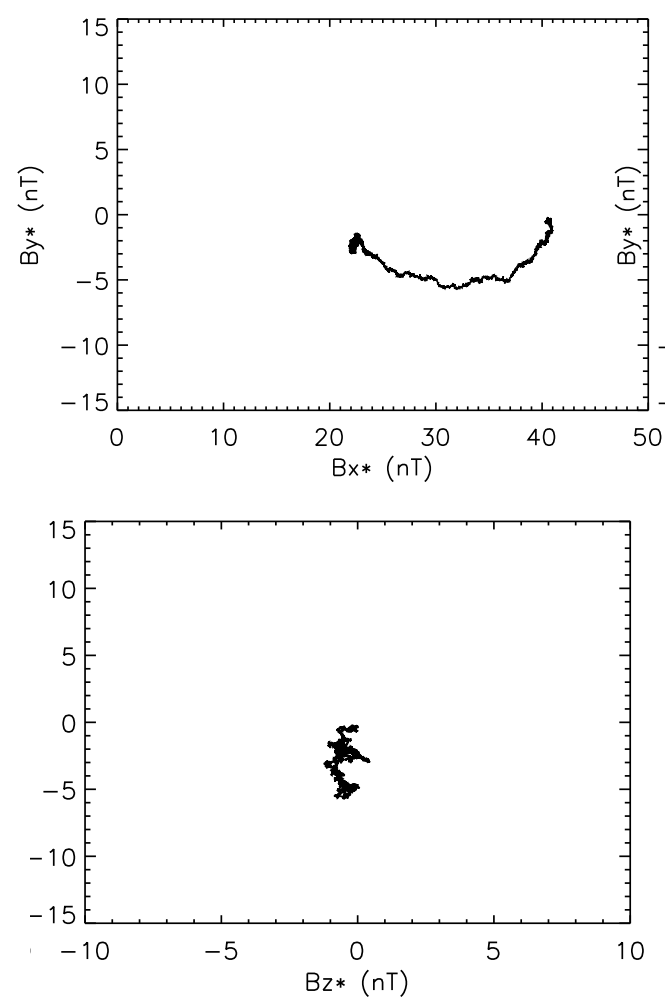

Fig. 6. Magnetic field rotation in the plane of maximum variance and in the plane of minimum variance during an SI event observed on 17 August 2001.

the 64-s averaged magnetic field and plasma data from ACE. Figure 9 shows the solar wind dynamic pressure, the static pressure and $S$ for the 17 August 2001 shock. $S$ decreases across the shock, suggesting that the tail flaring decreases. For all events a similar, clear decrease in the $S$ parameter was observed. The relationship between $\Delta P_{\text {dyn }}^{S W}$ and $\Delta B$ in Fig. 8a is better described by assuming a decrease in flaring angle $\alpha$ ( $5^{\circ}$ for the solid curve) than assuming constant flaring.

Figure $8 \mathrm{~b}$ presents the comparison between the shock/solar wind speed and the duration of eight tail lobe SIs. The 25 October 2001 event was excluded because during the magnetic field increase Cluster moved from the lobe to the magnetospheric boundary layer (see Sect. 5). The transition scale length is shorter closer to the tail boundary than near the axis (Fig. 5a). The events observed when Cluster was located more than $10 R_{E}$ from the $X$-axis are shown by circles. Figure $8 \mathrm{~b}$ shows rather weak anticorrelation (correlation coefficient -0.62 ).

\section{The tail compression (TC) model}

\subsection{Model description}

Collier et al. (1998) showed that a Cartesian 2-D model, assuming a uniform magnetic field and a step-like 

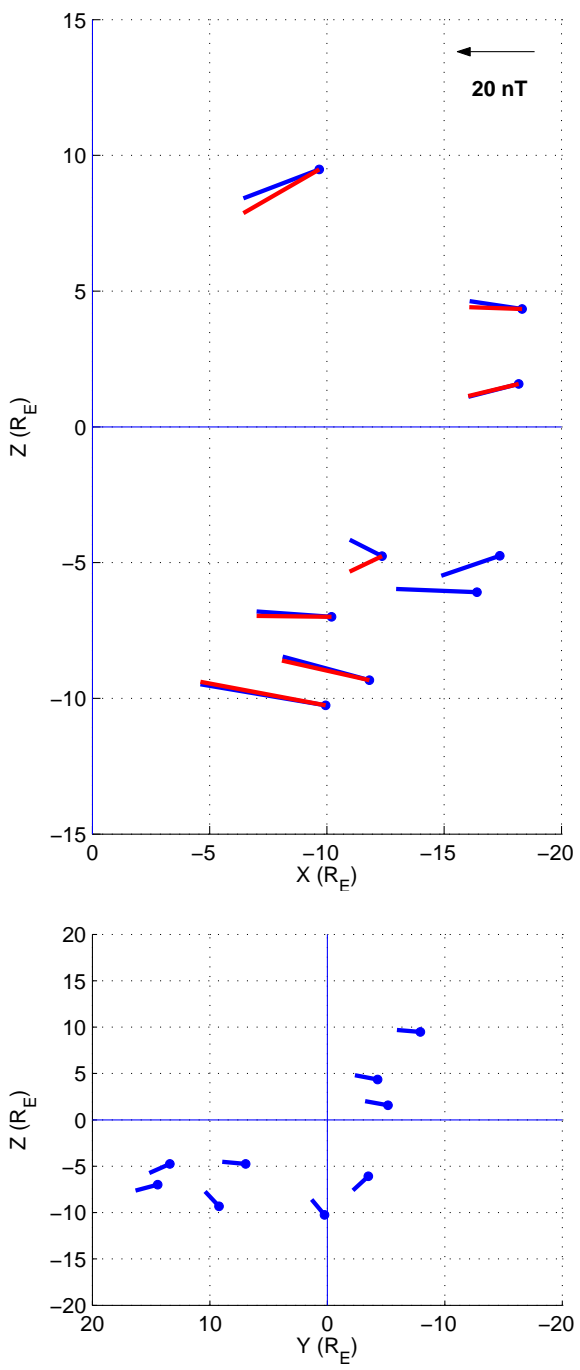

Fig. 7. (left) Locations of the maximum variance vectors $\left(\hat{\mathbf{e}}_{\mathbf{x}}^{*}\right)$ projected onto the GSM XY plane (blue). The root of the vector has been placed at the spacecraft location at the time of the event. The length of the each vector represents the unperturbed magnetic field magnitude, normalized to the scale shown by the arrow. Circles show the spacecraft position at the time of the SI event. Red lines show the projected shock normal direction for each SI event that was associated with a shock. Note that the shock normal directions are defined to point in the direction of the upstream solar wind. (right) Unit intermediate variance vectors $\hat{\mathbf{e}_{\mathbf{x}}}{ }^{*}$ projected in the GSM YZ plane. Circles show the spacecraft position at the time of the SI event.

compression, can accurately describe the magnetic field profile and characteristic time scales for tail lobe SIs. We have applied this "tail compression" (TC) model to our SI events. We briefly summarize the model here. The tail lobe is described by a vacuum (motivated by the very low density in the lobes), current-free asymptotically constant magnetic field constrained between two walls (see Fig. 5). The Alfvén speed is assumed infinite, and the magnetotail remains in equilibrium as the pressure enhancement propagates downtail. The $X$-axis is aligned with the tail axis and the
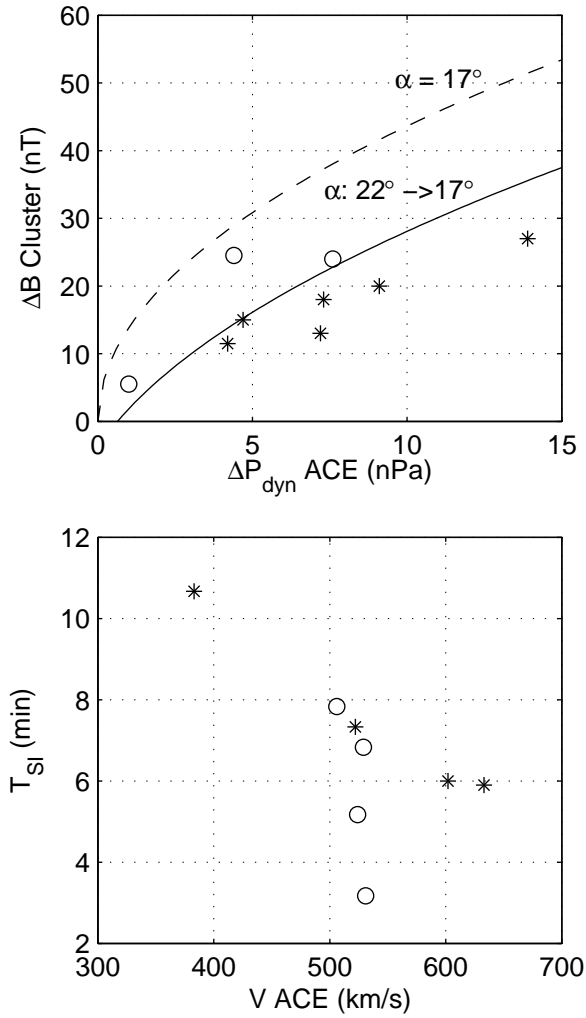

Fig. 8. Changes in the solar wind dynamic pressure at ACE compared to the corresponding tail lobe SI amplitude at Cluster. The circles show the events during which Cluster was closest to the Earth $\left(\sim 10 R_{E}\right)$. For the dynamic pressure upstream of the shock the value of $1 \mathrm{nPa}$ has been used (a). The propagation speed of the shock or solar wind speed compared to the duration time of the tail lobe SI (b).

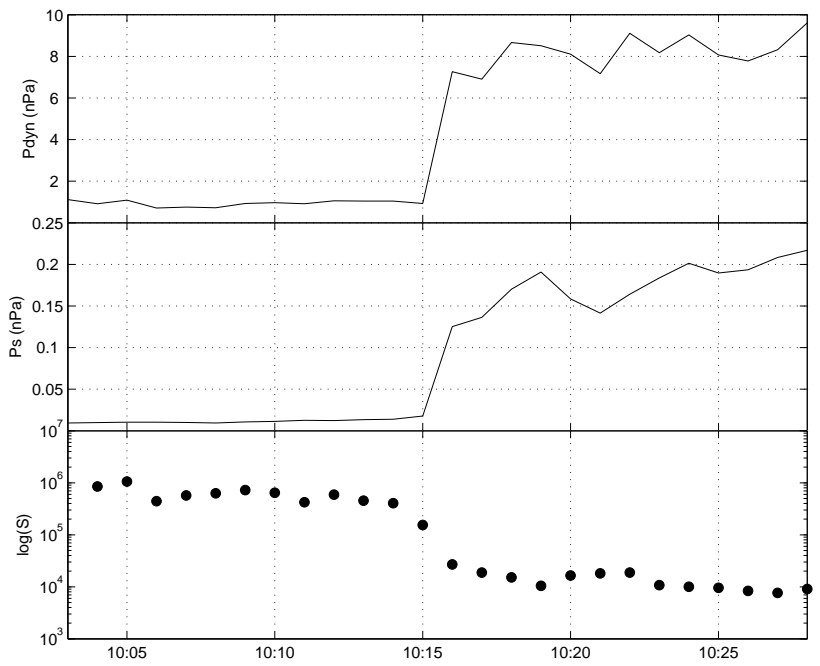

Fig. 9. Solar wind dynamic pressure, static pressure, and the $S$ parameter (plotted logarithmically) for an interplanetary shock observed on 17 August 2001. Note the different scales in panels (a) and (b) showing dynamic and static pressures, respectively. 


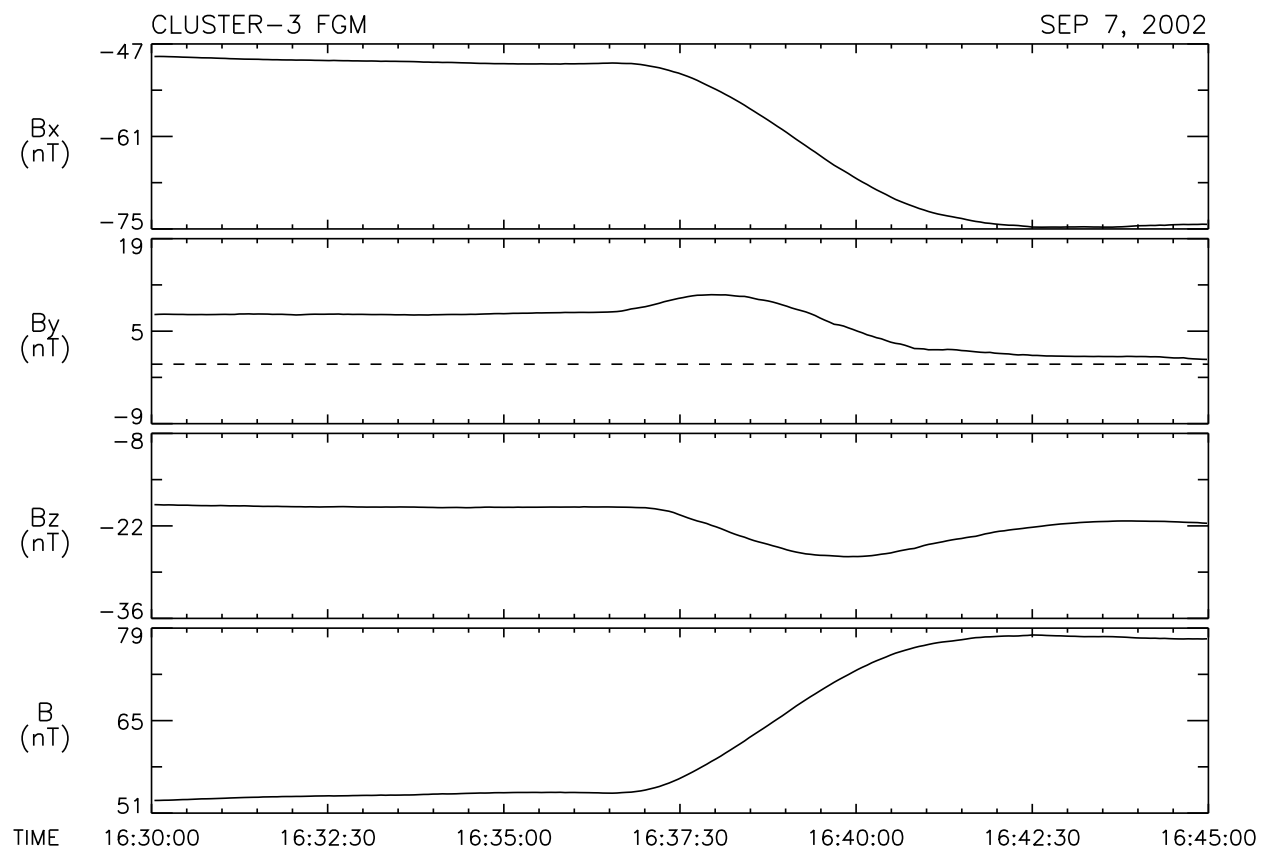

Fig. 10. Tail lobe SI event on 7 September 2002. Panels show magnetic field components in the GSM coordinate system and the magnetic field magnitudes. Cluster was located at a GSM position of $(-9.95,0.23,-10.26) R_{E}$.

$Y$-axis is transverse to the tail axis. The increase in the solar wind dynamic pressure decreases the tail width from $R$ to $R_{2}=R-\Delta R$, where $\Delta \ll 1$. The lobe field increases from $B_{0}$ to $B_{0}(1+\Delta)$ when flux conservation through a 1-D surface is assumed. Changes in the $X$ and $Y$ components of the magnetic fields are:

$$
\begin{aligned}
& B_{x}=B_{0}\left(1+\frac{\Delta}{2} \frac{\exp (\pi x / R)+\cos (\pi y / R)}{\cosh (\pi x / R)+\cos (\pi y / R)}\right), \\
& B_{y}=-B_{0}\left(\frac{\Delta}{2} \frac{\sin (\pi y / R)}{\cosh (\pi x / R)+\cos (\pi y / R)}\right) .
\end{aligned}
$$

$B_{x}$ makes a quick transition between the asymptotic states while $B_{y}$ exhibits a positive or negative deflection. As illustrated in Fig. 5a the direction of the $B_{y}$ deflection depends on the position of Cluster in the magnetosphere. Table 2 shows that the directions of the $B_{y}$ deflections were as expected based on the spacecraft location at that time. The peak of the $B_{y}$ transition occurs at the time of the mid-point of the $B_{x}$ transition (at $x=0)$. At the tail axis $(y / R=0) B_{y}$ remains unchanged and the amplitude of the deflection increases when the boundary is approach. The model is also applicable to other planes as well. Far from the XY-plane the deflection is seen mainly in the $Z$ component. As demonstrated by Fig. $5 \mathrm{~b}$ the direction of the $B_{z}$ deflection is always negative.

Figure 10 shows Cluster magnetic field measurements on 17 September 2002 when Cluster was located much closer to the magnetopause in the GSM $Z$-direction than in the GSM $Y$-direction. The amplitude of the $B_{y}$ and $B_{z}$ deflections are measured from the unperturbed value before the SI compression to the peak of the deflection. For this event the deflection in $B_{z}$ is clearly larger $\left(\left|\Delta B_{z}\right|=9 \mathrm{nT}\right)$ than the deflection in $B_{y}$ $\left(\left|\Delta B_{y}\right|=1.5 \mathrm{nT}\right)$. Table 2 shows that the amplitude of the $B_{y}$ deflection was larger than the amplitude of the $B_{z}$ deflection when Cluster had comparable GSM $Y$ and $Z$ positions (i.e. events 1, 3, 7 and 9). At the time of the SI event on 11 October 2001, Cluster was located at about the same distance from the $X$-axis in the GSM $Y$ and $Z$ directions (Fig. 11). For this event the $\left|\Delta B_{y}\right|$ was $15 \mathrm{nT}$ and the $\left|\Delta B_{z}\right| 8 \mathrm{nT}$. This suggests that the magnitude of the magnetospheric compression is different in the $Y$ and $Z$ directions. Figure 6 supports this same conclusion by demonstrating that the intermediate variance vectors for SI events are more aligned with the GSM $Y$-direction.

\subsection{Magnetic field increase}

Figure 12 shows the ratio of the final tail lobe magnetic field magnitude divided by the initial magnetic field magnitude $\left(B_{2} / B_{0}\right)$ as a function of the $\Delta$-parameter $\left(\Delta=1-R_{2} / R\right)$. The blue solid line indicates the prediction according to the 2-D Collier et al. (1998) model, i.e. $B_{2} / B_{0}=(1+\Delta)$. The red dashed line represents the case were the magnetosphere with a circular crosssection is symmetrically compressed, assuming the conservation of the magnetic flux:

$\frac{B_{2}}{B_{0}}=\left(\frac{R_{0}}{R_{2}}\right)^{2}=\frac{1}{(1-\Delta)^{2}}$.

In the case of a small $\Delta$ we obtain from Eq. (6) $B_{2} / B_{0} \approx(1+2 \Delta)$, that is shown by the red dash-dot line in Fig. 12. Stars and circles indicate tail lobe SI events in this study. To estimate the $\Delta$-parameter we have calculated the 


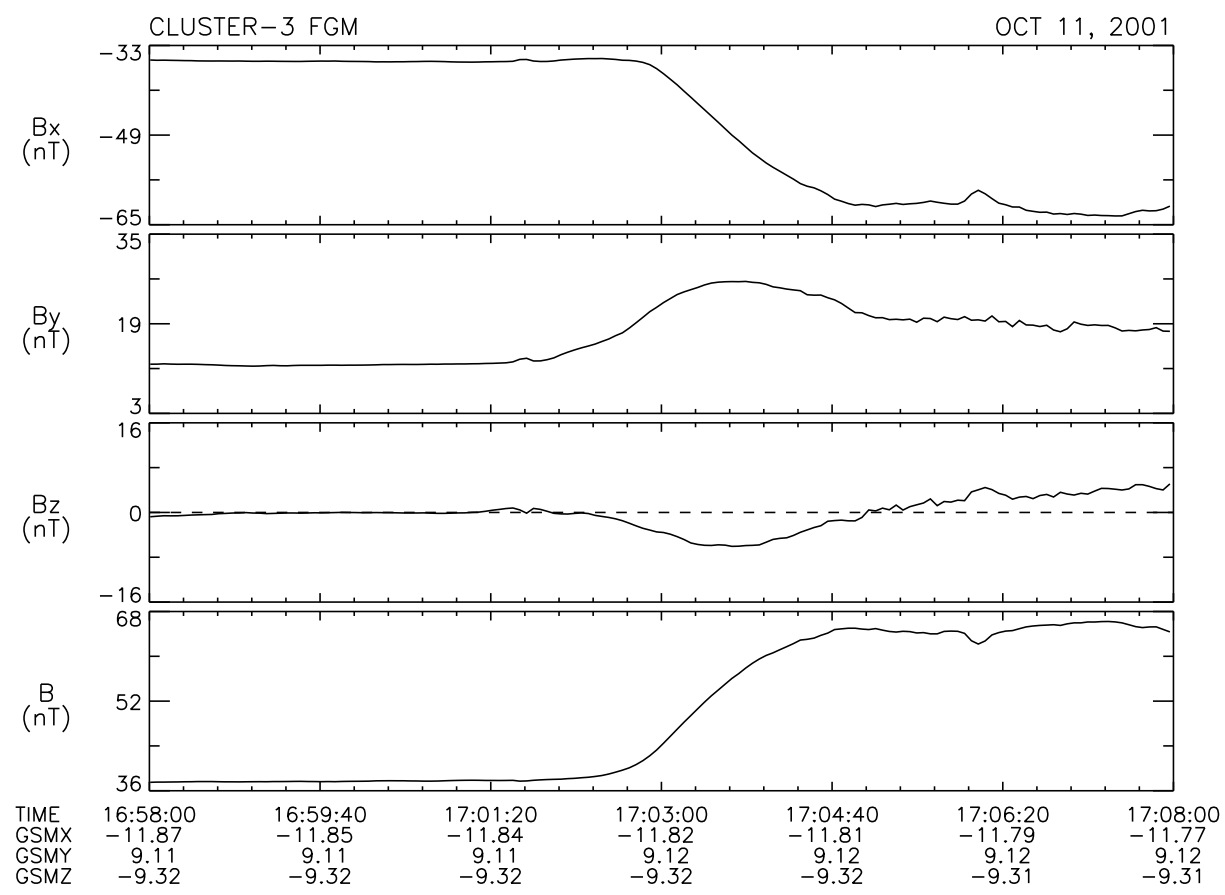

Fig. 11. Tail lobe SI event on 11 October 2001. Panels show magnetic field components in the GSM coordinate system and the magnetic field magnitude. The GSM-position of Cluster was $(-11.81,9.12,-9.33) R_{E}$.

tail radius before $(R)$ and after $\left(R_{2}\right)$ the compression using the Shue et al. (1998) model (black stars) and the Petrinec and Russell (1996) model (green circles). Both models depend on the IMF $Z$ component and solar wind dynamic pressure. For southward IMF the Petrinec and Russell (1996) model produces less tail flaring than the Shue et al. (1998) model. We used 20-min averaged solar wind values upstream and downstream of the shock as input to the models. The diamonds show the two tail lobe SI events presented in Collier et al. (1998) (on 9 March 1995 and on 17 August 1995).

The Petrinec and Russell (1996) model gives a larger $\Delta$ parameter than the Shue et al. (1998) model. Nearly all data points in Fig. 12 for which the Shue et al. (1998) model was used are above the 3-D model curve. When the Petrinec and Russell (1996) model is used data points fall between the 3-D model curve and the Collier et al. (1998) model prediction.

Being 2-D, the Collier et al. (1998) model naturally underestimates the increase in the magnetic field magnitude. However, the behavior of the magnetic field $X$ component is very accurately described by the model using a standard least-squares fit of Eq. (4) to the data (see Collier et al. (1998) and the following section). If we multiply $\Delta$ in Eq. (4) by a factor of two, the asymptotic magnetic field magnitude becomes $(1+2 \Delta) \times B_{0}$. This is the same as the asymptotic value in the 3-D compression expressed by Eq. (6) in the limit of small $\Delta$. If we assume that values of $\Delta$ estimated using the Petrinec and Russell (1996) model (green dots) describe accurately the true magnetospheric compression, the data points in Fig. 12 fit best around the model curve for the 3 -D compression in the limit of small $\Delta$. Thus, the Collier et

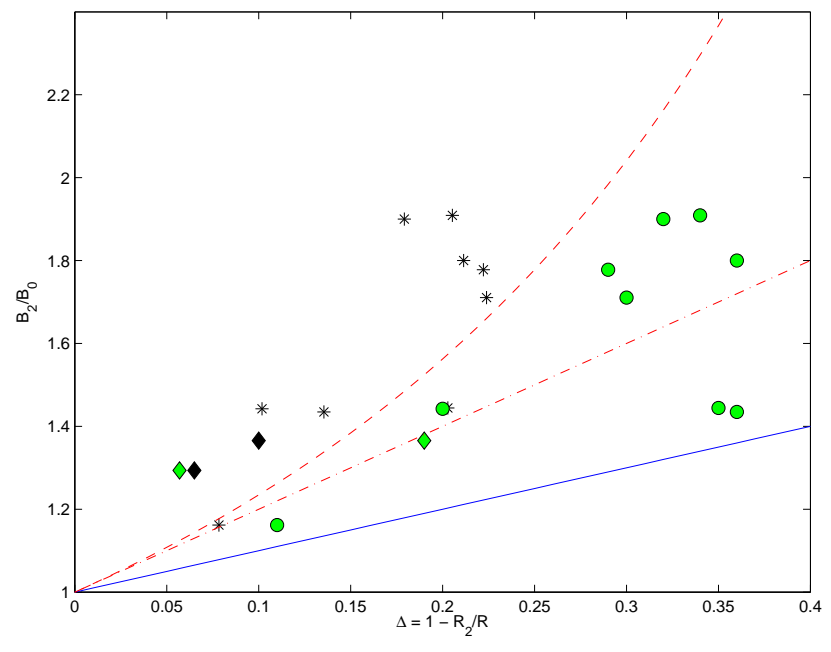

Fig. 12. Theoretical curves for the ratio of the final and initial magnetic field magnitudes as a function of $\Delta$ in the 2-D Collier et al. (1998) model (blue solid line), in 3-D compression, red dashed line, see Eq. (6), and in the case of small $\Delta$ for 3-D compression (red dash-dot line). Data points show tail lobe SI events: Stars and circles show the events in this study where we have used the Shue et al. (1998) model and the Petrinec and Russell (1996) model, respectively, to approximate $\Delta$. Diamonds show two events presented in Collier et al. (1998).

al. (1998) model, with the additional factor of 2 to mimic the behaviour of the 3-D model with cylindrical geometry appears to describe the data better than the original 2-D form, see Eqs. (4) and (5). 
Table 3. Results from the fit of the magnetic field $X$-component to Eq. (7). $\theta$ is calculated from Eq. (11). $B_{0}$ is the initial asymptotic value of the magnetic field, $B_{1}$ the increase of the magnetic field, $t_{0}$ the midpoint of the rise, $\tau$ the characteristic time scale from the fit and $\tau_{i}$ the initial guess according to Eq. (9).

\begin{tabular}{llllcll}
\hline $\mathrm{N}$ & $\begin{array}{l}\theta \\
\left({ }^{\circ}\right)\end{array}$ & $\begin{array}{l}B_{0} \\
(\mathrm{nT})\end{array}$ & $\begin{array}{l}B_{1} \\
(\mathrm{nT})\end{array}$ & $\begin{array}{c}t_{0} \\
(\mathrm{~h})\end{array}$ & $\begin{array}{l}\tau \\
(\mathrm{h})\end{array}$ & $\begin{array}{l}\tau_{i} \\
(\mathrm{~h})\end{array}$ \\
\hline 1 & 0.73 & 22.3 & 18.7 & 11.115861 & 0.017 & 0.025 \\
2 & 1.01 & 24.8 & 11.6 & 9.691994 & 0.015 & 0.019 \\
3 & 1.34 & 37.2 & 27.7 & 17.058411 & 0.010 & 0.021 \\
4 & & & & & & \\
5 & 1.13 & 30.6 & 24.5 & 3.3726585 & 0.016 & 0.023 \\
6 & & & & & & \\
7 & 1.10 & 31.8 & 5.9 & 13.668289 & 0.021 & 0.022 \\
8 & 0.77 & 20.2 & 18.6 & 18.820975 & 0.018 & 0.020 \\
9 & 0.60 & 30.7 & 16.6 & 11.590268 & 0.027 & 0.032 \\
10 & 0.86 & 48.3 & 26.2 & 16.654298 & 0.014 & 0.022 \\
\hline
\end{tabular}
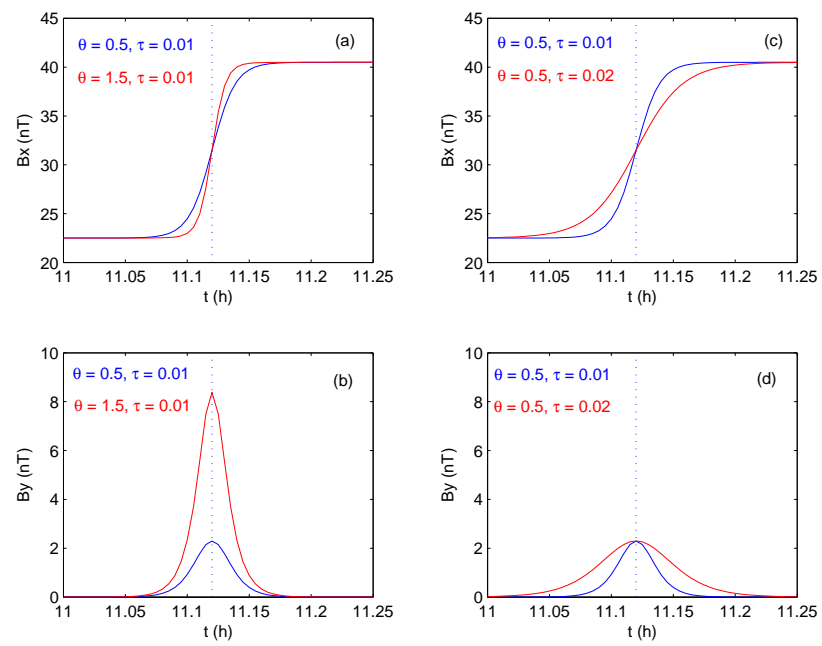

Fig. 13. Changes in $B_{x}$ and $B_{y}$ components according to the Collier et al. (1998) model for different values of $\tau$ and $\theta$. The dashed line shows the midpoint time $t_{0}$.

A larger than predicted increase in the tail lobe magnetic field may result from flux loading into the tail lobes due to reconnection at the dayside magnetopause. Also, changes in the solar wind $V_{y}$ and $V_{z}$ components at the shock can deflect the magnetotail so that after the shock passage the spacecraft position relative to the tail axis may have changed, whereas the model assumes it has not.

\subsection{Fitting procedure}

The $X$-coordinate in Eqs. (4) and (5) can be replaced by $v\left(t-t_{0}\right)$, assuming that the pressure discontinuity that causes the constriction moves down the tail with speed $v ; t_{0}$ is the time at which the discontinuity passes the spacecraft location. In order to take into consideration possible deflection of the magnetotail and that in some of the events the compression was observed primarily in the $B_{z}$ component, the fitting was performed in a minimum variance coordinate system (Sonnerup and Cahill, 1967). From Eqs. (4) and (5) we obtain the functional forms:

$$
\begin{aligned}
& B_{x}^{*}=B_{0}+B_{1} \times \frac{1+e^{-\left(t-t_{0}\right) / \tau} \cos \theta}{1+e^{-2\left(t-t_{0}\right) / \tau}+2 e^{-\left(t-t_{0}\right) / \tau} \cos \theta}, \\
& B_{y}^{*}=-B_{1} \times \frac{\sin \theta}{1+e^{-2\left(t-t_{0}\right) / \tau}+2 e^{-\left(t-t_{0}\right) / \tau} \cos \theta},
\end{aligned}
$$

where $B_{1}=B_{0} \Delta$ is the magnitude of the change in $B_{x}^{*}$. The characteristic time scale of the transition $\tau$ depends on the magnetotail width $R$ and solar wind speed. The $\theta$-parameter depends on the ratio of the Cluster $Y$ component to the tail width $R$ :

$$
\begin{aligned}
\tau & =\frac{R}{\pi v}, \\
\theta & =\frac{\pi y}{R} .
\end{aligned}
$$

Free parameters in the model are $B_{0}, B_{1}, t_{0}, \tau$ and $\theta$. Initial values of $B_{0}, B_{1}$ and $t_{0}$ for the fitting are estimated from the Cluster magnetic field data. Figure 13 shows examples of the changes in $B_{x}$ and $B_{y}$ for different values of $\theta$ and $\tau$. Increasing $\tau$ and decreasing $\theta$ increases the transition time of $B_{x}$ between the asymptotic values. Small changes in $\tau$ have a larger affect on the $B_{x}$ profile than small changes in $\theta$. Thus, we can justify using a fixed $\theta$. Increasing $\tau$ makes the deflection in the $Y$ component wider, but the peak value is not changed. Increasing $\theta$ widens the deflection in the $Y$ component, but also increases its peak magnitude, as shown in Fig. 13b. The $\theta$ parameter can be calculated from Eq. (8) at $t=t_{0}$, and $B_{1}$ and $B_{y}^{*}$ are determined from the Cluster data. Solving $\theta$ from Eq. (8) gives:

$\theta=\cos ^{-1}\left(\frac{-4 B_{y}^{* 2} \pm \sqrt{16 B_{y}^{* 4}+B_{1}^{4}}}{4 B_{y}^{* 2}+B_{1}^{2}}.\right)$

We performed a standard least-squares fit using Eq. (7) to the Cluster data using a fixed $\theta$ evaluated from Eq. (11). The results are shown in Table 3. In the 6 November 2001 event (number 6) dramatic changes in the magnetic field components resulted from the magnetopause moving past Cluster. For the 25 October 2001 event, we did not perform the fit because the magnetic field behavior was too irregular due to Cluster's transition into the magnetospheric boundary layer.

Figures 14 and 15 show magnetic field $B_{x}^{*}$ and $B_{y}^{*}$ profiles and the result of the fit to the Cluster $\mathrm{C} 3$ data during 17 August 2001 and 26 August 2002 SI events, respectively. In both cases the transition of the $B_{x}^{*}$ component is wellcaptured by the model. Note that we did not perform a leastsquares fit to the magnetic field $Y$ component. The modeled $B_{y}^{*}$-curve was obtained by using the fit parameters from the $B_{x}^{*}$ modeling fed into Eq. (8). The amplitude of the deflection 

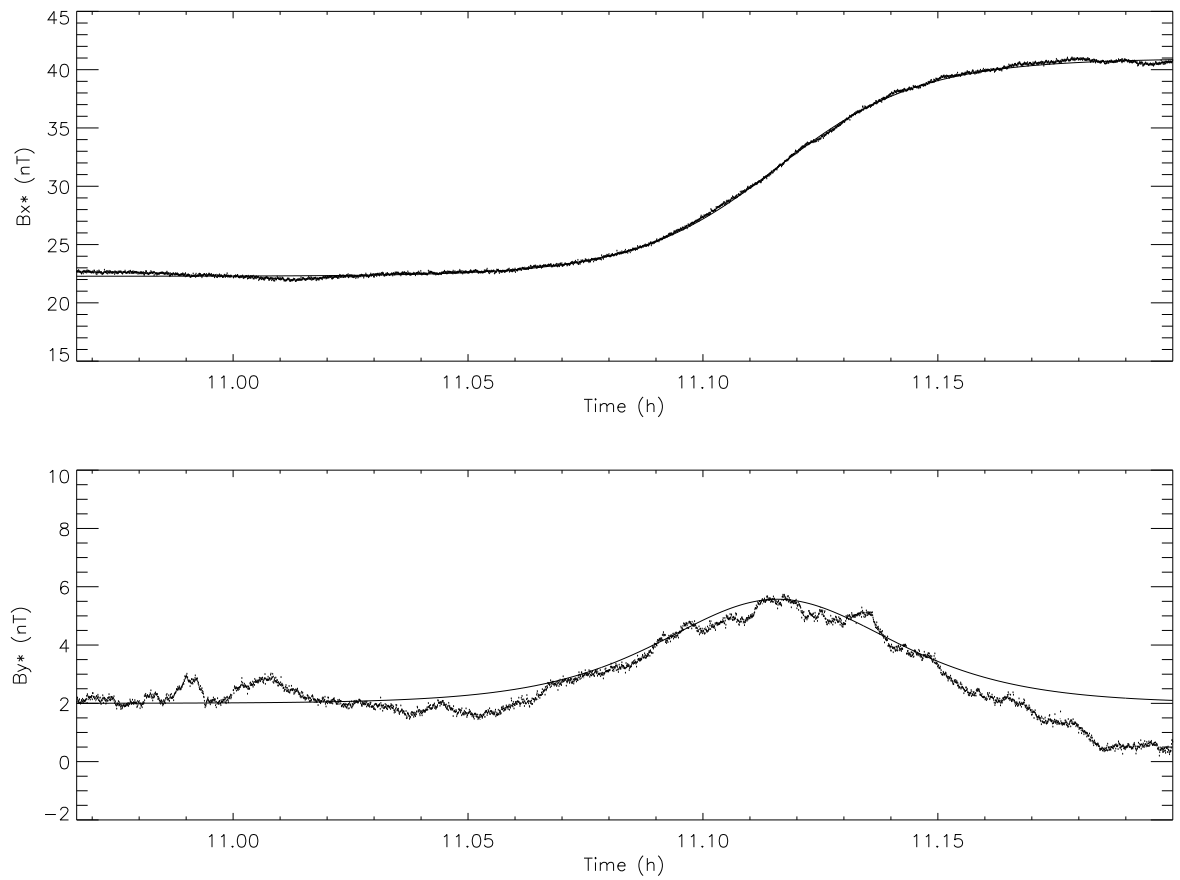

Fig. 14. Results of the fit of Eq. (7) to the magnetic field maximum variance component $B_{x}^{*}$ measured by Cluster C3 for an SI event on 17 August 2001. The solid line in the panel showing the intermediate variance component, $B_{y}^{*}$, is not a fit but the profile described by Eq. (8) using the parameters determined by the fit to the maximum variance component in the upper panel.

Table 4. Timing considerations between WIND-Cluster (W-C) and Geotail-Cluster (G-C). The table shows the event number, time of the shock/pressure jump at Wind $\left(T_{S W}\right)$ and at Geotail $\left(T_{S G}\right)$, observed and calculated times of the tail lobe SI at Cluster $\left(t_{W-C}^{\mathrm{obs}}, t_{W-C}^{\mathrm{calc}}, t_{G-C}^{\mathrm{obs}}, t_{G-C}^{\mathrm{calc}}\right)$, the differences between the observed and calculated times $\left(\Delta t_{W-C}, \Delta t_{G-C}\right)$ and the percentage difference between the calculated and observed times.

\begin{tabular}{lrrrrrrrrrr}
\hline $\mathrm{N}$ & $\begin{array}{r}T_{S W} \\
(\mathrm{UT})\end{array}$ & $\begin{array}{r}t_{W-C}^{\mathrm{obs}}(\mathrm{min}) \\
(\mathrm{min})\end{array}$ & $\begin{array}{r}t_{W-C}^{\mathrm{calc}} \\
(\mathrm{min})\end{array}$ & $\begin{array}{r}\Delta t_{W-C} \\
(\min )\end{array}$ & & $\begin{array}{r}T_{S G} \\
(\mathrm{UT})\end{array}$ & $\begin{array}{r}t_{G-C}^{\mathrm{obs}} \\
(\min )\end{array}$ & $\begin{array}{r}t_{G-C}^{\mathrm{calc}} \\
(\min )\end{array}$ & $\begin{array}{r}\Delta t_{G-C} \\
(\min )\end{array}$ \\
\hline 1 & $11: 01: 43$ & 5.37 & 5.18 & +0.19 & $+3.5 \%$ & $11: 00: 22$ & 6.44 & 6.58 & -0.14 & $-2.1 \%$ \\
2 & $9: 29: 28$ & 14.32 & 12.00 & +2.32 & $+5.6 \%$ & $9: 38: 00$ & 4.80 & 3.47 & +1.33 & $+27.7 \%$ \\
3 & $16: 50: 57$ & 11.99 & 12.55 & -0.56 & $-4.7 \%$ & $16: 59: 17$ & 3.77 & 4.22 & -0.45 & $-12 \%$ \\
4 & $08: 58: 00$ & 3.09 & 6.17 & -6.17 & $-99.0 \%$ & $08: 47: 14$ & 6.10 & 4.60 & +1.5 & $+24.6 \%$ \\
5 & $3: 13: 50$ & 8.85 & 8.55 & +0.3 & $+3.4 \%$ & $03: 14: 40$ & 7.72 & 7.75 & -0.03 & $-0.39 \%$ \\
6 & $01: 54: 26$ & & & & & & & & & $-0.4 \%$ \\
7 & $13: 29: 17$ & 11.08 & 10.81 & +0.27 & $+2.5 \%$ & $13: 33: 02$ & 6.67 & 7.07 & -0.4 & $-6.0 \%$ \\
8 & $18: 40: 50$ & 8.97 & 8.5 & +0.47 & $+5.2 \%$ & $18: 49: 00$ & 0.97 & 0.6 & +0.37 & $-38 \%$ \\
9 & $11: 16: 00$ & & & & & $11: 24: 03$ & 12.19 & 11.37 & +0.82 & $+6.7 \%$ \\
10 & $16: 22: 10$ & 16.58 & 16.08 & +0.50 & $+3.0 \%$ & $16: 33: 31$ & 4.88 & 4.73 & +0.15 & $+3.1 \%$ \\
\hline
\end{tabular}

in $B_{y}^{*}$ matches the data because the $\theta$ parameter used in the fit for $B_{x}^{*}$ was determined from the $B_{y}^{*}$ data using Eq. (11). The width of the profile, which is determined by the $B_{x}^{*}$ fitting, is well described by Eq. (8). We performed this analysis on all events shown in Table 3 and in every case, the fitted curves showed agreement with the data that was as good as that shown in Figs. (14) and (15).

\section{Timing considerations}

\subsection{Event timing}

We also performed a timing analysis between the shock/pressure increase (using 3-s magnetic field data) at Wind/Geotail and the SI event at Cluster. Figure 5 demonstrates that large compressions cause changes in the tail lobe magnetic field significantly before the actual pressure enhancement has reached the location of the spacecraft, as field 

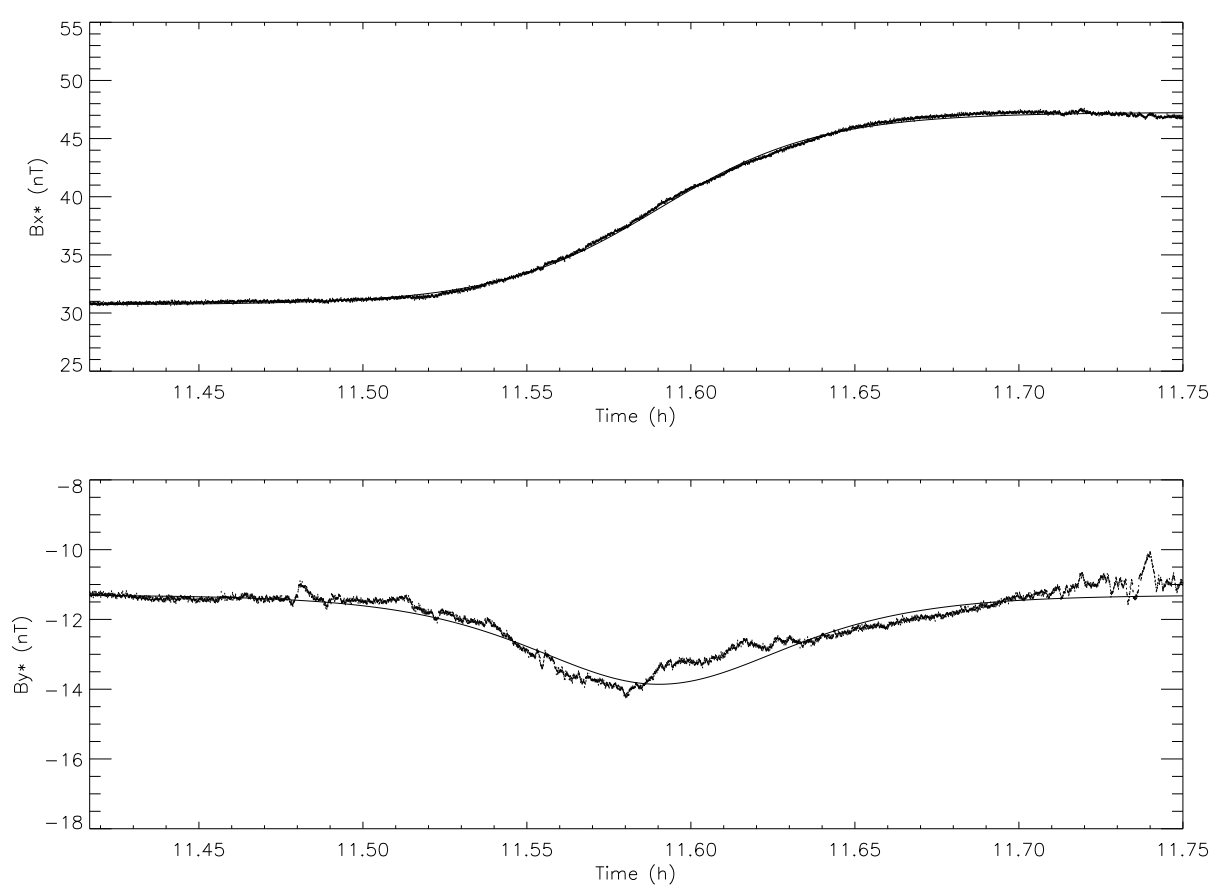

Fig. 15. The fit results for an SI event on 26 August 2002.

Table 5. Estimated propagation speeds of the disturbances using timing between Cluster spacecraft pairs. Columns are the event number, spacecraft pair used (S/C), separation of spacecraft in the $X$ and $Y$ directions, the propagation speed calculated using time delays between two Cluster satellites $\left(V_{\text {prog }}\right)$, the propagation speed calculated using the correlation analysis $\left(V_{\text {corr }}\right)$, the difference between the speeds obtained using the two methods (diff) and the propagation speed of a shock or pressure discontinuity $\left(V_{S}\right)$.

\begin{tabular}{llllllll}
\hline $\mathrm{N}$ & S/C pair & $|\Delta X|$ & $\begin{array}{l}|\Delta Y| \\
\left(R_{E}\right)\end{array}$ & $\begin{array}{l}V_{\text {prog }} \\
\left(R_{E}\right)\end{array}$ & $\begin{array}{l}V_{\text {corr }} \\
\left(\mathrm{km} / \mathrm{s}^{-1}\right)\end{array}$ & $\begin{array}{l}\text { diff } \\
\left(\mathrm{km} / \mathrm{s}^{-1}\right)\end{array}$ & $\begin{array}{l}V_{S} \\
\left(\mathrm{~km} / \mathrm{s}^{-1}\right)\end{array}$ \\
\hline 1 & C1-C4 & 0.27 & 0.11 & 507 & 309 & 198 & 522 \\
2 & C1-C4 & 0.24 & 0.11 & 295 & 637 & -342 & 633 \\
3 & C2-C4 & 0.30 & 0.08 & 720 & 573 & 147 & 531 \\
4 & no fit & & & & & & \\
5 & C1-C4 & 0.21 & 0.23 & 501 & 446 & 55 & 529 \\
6 & no fit & & & & & & \\
7 & C1-C4 & 0.61 & 0.39 & 628 & 417 & 211 & 506 \\
8 & & & & & & & \\
9 & C1-C4 & 1.10 & 0.07 & 365 & 417 & -52 & 383 \\
10 & C2-C3 & 0.62 & 0.21 & 878 & - & & 524 \\
\hline
\end{tabular}

lines have to bend to fit into the constricted region (Collier et al., 1998). Thus, the midpoint time of the SI transition $\left(t_{0}\right)$ has been used instead of the start time. We approximated the shock as a plane moving with a uniform velocity in the direction of its normal. Events 2 and 9 were not associated with shocks, so we assumed that the pressure enhancement was propagating at the solar wind speed. For event 6 , solar wind plasma data were not available from Wind (no ACE plasma data were available either) and Geotail was located inside the magnetosphere. Table 4 shows the results of the timing analysis. Time differences obtained from the Geotail-Cluster pair should be more reliable, as in general, Geotail was lo- cated closer to the Earth and had a smaller distance from the Sun-Earth line than WIND. For event 2, for which we could not determine the shock orientation, the differences between the calculated and observed values were large. Also, for the event 4 the difference was several minutes. In this case the duration of the tail lobe SI given in Table 2 may be too short by a few minutes, because Cluster moved from the lobe to the magnetospheric boundary layer before the magnetic field components had completed their full transitions. Otherwise, the differences between the calculated and observed times using the Geotail- Cluster pair are less than $30 \mathrm{~s}$. This is consistent with the assumption that the midpoint of the 
compression should be observed at the spacecraft about the time when the pressure discontinuity moves past the spacecraft.

4.2 The disturbance propagation speed using time delays between Cluster spacecraft

The multi-spacecraft Cluster mission allows us to determine the propagation speed of the disturbance between the closelyspaced Cluster spacecraft. In Table 3 we summarize the results from the fitting of the $\mathrm{C} 3$ data. For each event we performed the fitting for all four Cluster spacecraft. We calculated the propagation speed of the disturbance between the spacecraft using the midpoint times of the tail lobe SIs and the separation of spacecraft in the $X$ direction. Table 5 shows the calculated propagation speed of the disturbance between one satellite pair for each event. If the high resolution data was available, we used the satellite pair $\mathrm{C} 1$ and $\mathrm{C} 4$ because they had the largest separation in the $X$ direction. For the 18 August 2002 event the fit was performed only for one spacecraft because, as mentioned earlier, the magnetic field behavior was rather irregular. The inferred speeds vary between $\sim 300 \mathrm{~km} / \mathrm{s}^{-1}$ and $900 \mathrm{~km} /$ and are not apparently correlated with the speed of the associated shock or solar wind speed. The reason for this may be non-radial propagation of the disturbance. In any case, they are clearly of the order of the solar wind speed rather than of the order of the Alfvén speed in the tail lobes $\left(>1000 \mathrm{~km} / \mathrm{s}^{-1}\right)$, as in the scenario suggested by Sugiura et al. (1968).

The panel $V_{\text {corr }}$ in Table 5 gives the propagation speed of the disturbance estimated using the correlation calculation between the $B_{x}$ measurements from two Cluster spacecraft. The results are given for the same spacecraft pairs that were used in the previous section. We performed a time lagged cross-correlation between the GSM $B_{x}$ component of the magnetic field measured at two spacecraft pairs. The propagation speed of the disturbance from one spacecraft to the other is $V_{\text {corr }}=\left(X_{1}-X_{2}\right) / \Delta T_{12}$, where $\Delta T_{12}$ is the lag time. For the 25 July 2002 and 26 August 2002 events the speeds obtained using the two methods are nearly the same, but for the other events differences exceed $100 \mathrm{~km} / \mathrm{s}^{-1}$.

\section{Events with transition close to the magnetopause}

During three tail lobe SI events (11 October 2001; 25 October 2001 and 6 November 2001) Cluster was located close to the tail boundary. For the first two events, Cluster observed the inward moving magnetopause, and the plasma and magnetic field signatures typical of the magnetospheric boundary layer. For the third event Cluster detected a magnetopause crossing.

Figure 16 shows magnetic field and plasma data during the SI event on 25 October 2001. From top to bottom, the panels give magnetic field magnitude, magnetic field components in the GSM coordinate system, plasma density, velocity, plasma beta, and proton flux. At this time Cluster was lo-

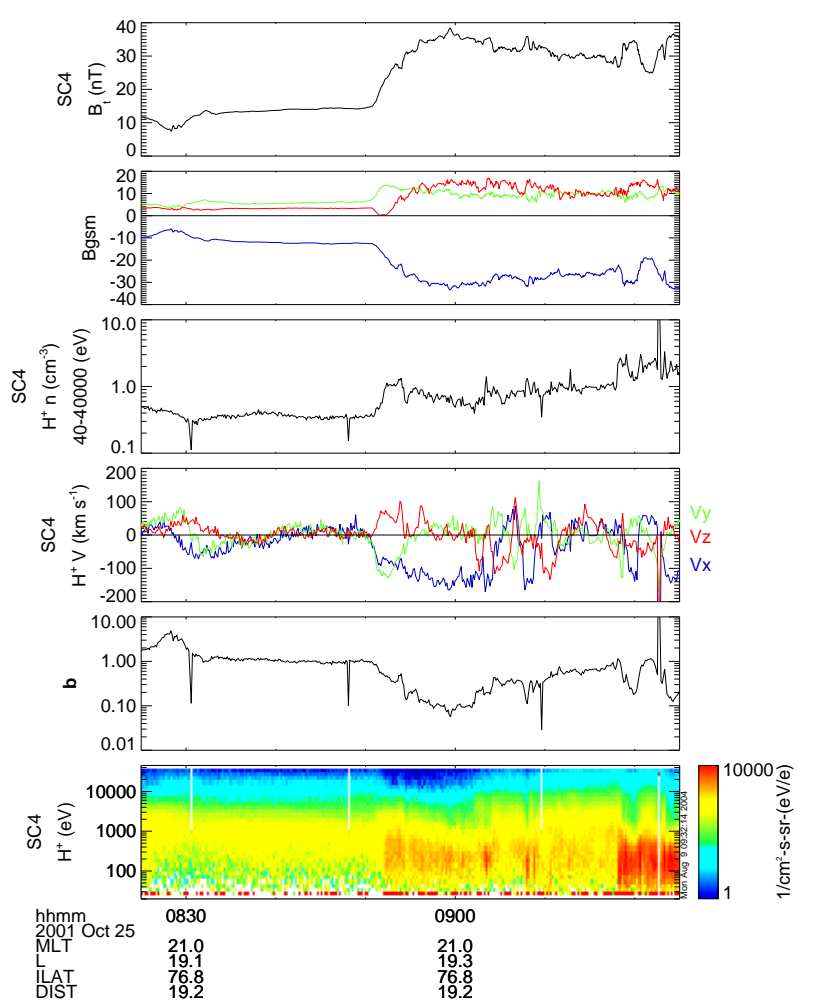

Fig. 16. Cluster FMG and CIS data during an SI event observed on 25 October 2001. The panels show magnetic field magnitude, magnetic field GSM components ( $B_{x}$ : blue, $B_{y}$ : green, $B_{z}:$ red), $H^{+}$density, components of the velocity, plasma beta and the $H^{+}$ flux.

cated in the southern tail lobe at a GSM position of $(-12.53$, $13.4,-4.76) R_{E}$ moving away from the plasma sheet. The proximity of the plasma sheet before the SI is inferred by the steady, but relatively weak magnetic field $X$ component and a plasma beta close to unity. During the SI event $B_{x}$ decreased from $-15 \mathrm{nT}$ to $-28.5 \mathrm{nT}$ between 08:50:30 and 08:51:55 UT, after which the magnetic field changes were more irregular. At this time there was an increase in the proton density and the proton flux, suggesting the magnetospheric boundary layer plasma with energies below $1 \mathrm{keV}$. A tailward plasma flow was observed for about 20 min after the SI onset. Short time scale perturbations were observed in the $V_{y}$ and $V_{z}$ components. The directions of the perturbations in $V_{y}$ were negative and in $V_{z}$ positive, showing that the magnetopause was moving towards Cluster. Using 20-min averaged solar wind values after the shock, the Shue et al. (1998) model predicts a tail radius of $17.4 R_{E}$, and the Petrinec and Russell (1996) model predicts a somewhat more compressed tail with a radius of $15.4 R_{E}$. In the YZ plane Cluster was located at a radial distance of $14.2 R_{E}$ from the origin. The solar wind $V_{y}$ had a value $\sim-30 \mathrm{~km} / \mathrm{s}^{-1}$ upstream of the shock that became slightly more negative downstream of the shock. This suggests that during the event the magnetotail was deflected so that Cluster was located even closer to the magnetopause boundary in the geocentric solar wind (GSW) 
system (where the $X$-axis is parallel to the solar wind flow) than in the GSM coordinate system.

At the time of the 11 October 2001 SI event (Fig. 11) Cluster was located in the southern tail lobe at the GSM position of $(-11.81,9.12,-9.33) R_{E}$. Like the previous event, the plasma and magnetic field data showed clear changes during this SI event. A tailward plasma flow lasting more than $1 \mathrm{~h}$ was observed starting at the time of the SI. The perturbations in $V_{y}$ (positive) and $V_{z}$ (negative) again demonstrate that the magnetopause was moving towards Cluster. Downstream of the shock, the Shue et al. (1998) model predicts a tail radius of $15.6 R_{E}$ and the Petrinec and Russell (1996) model predicts a tail radius of $14.4 R_{E}$. In the YZ-plane Cluster was located at a radial distance of $13.1 R_{E}$ from the origin. The solar wind $V_{y}$ had a value of $-45 \mathrm{~km} / \mathrm{s}^{-1}$ upstream of the shock that decreased to $-65 \mathrm{~km} / \mathrm{s}^{-1}$ after the shock, suggesting that Cluster was close to the magnetopause in GSW coordinates. This event has been analyzed in detail by Kim et al. (2004).

During the 6 November 2001 event, Cluster was located $15.1 R_{E}$ from the origin in the YZ-plane. We could not approximate the tail radius because the plasma data were not available. Anyhow, the compression may have been very large, as at the shock the magnetic field magnitude increased by almost $50 \mathrm{nT}$. Before the compression Cluster was located in the northern tail lobe close to the plasma sheet. Compared to the other events the transition was extremely rapid, lasting only about $4 \mathrm{~s}$. It seems likely that the magnetopause was compressed beyond the Cluster location and thus, after the transition Cluster was in the magnetosheath. Figure 17 presents Cluster FGM measurements during this event. Note that the plot covers only a 1-min time interval (01:54:0001:55:00 UT). From $B_{z}$ it is seen that $\mathrm{C} 2$ observed the transition first, then $\mathrm{C} 3$ and finally $\mathrm{C} 1$. The $\mathrm{C} 4$ high resolution data are not available for this event. Figure 18 displays the locations of Cluster spacecraft $\mathrm{C} 1, \mathrm{C} 2$ and $\mathrm{C} 4$ from the reference spacecraft $\mathrm{C} 3$. At the time of the transition $\mathrm{C} 2$ and C3 were located closest to the Earth so that $\mathrm{C} 3$ was only $0.01 R_{E}(\sim 64 \mathrm{~km})$ ahead of $\mathrm{C} 2$. However, $\mathrm{C} 2$ observed the transition $2.8 \mathrm{~s}$ earlier than $\mathrm{C} 3$. The separation between $\mathrm{C} 2$ and $\mathrm{C} 3$ in the GSM $Y$ directions was $\sim 0.20 R_{E}$ so that the observed time delay between the magnetopause crossings is probably due to the fact that $\mathrm{C} 2$ was located closer to the flanks of the magnetopause than $\mathrm{C} 3$. Using the difference in the GSM $Y$ positions and the time delay between $\mathrm{C} 2$ and $\mathrm{C} 3$ we obtained the speed $501 \mathrm{~km} / \mathrm{s}^{-1}$. C1 and C3 were nearly aligned in the GSM $X$ direction with a separation of $0.19 R_{E}$. The observed time delay was $1.96 \mathrm{~s}$, which gives a propagation speed of $618 \mathrm{~km} / \mathrm{s}^{-1}$. The propagation speed obtained using the spacecraft separation in the GSM $Y$ direction is smaller than the speed obtained using the separation in the GSM $X$ direction. The observations imply that the pressure enhancement did not cause a step-like compression, but rather a deformation in the magnetopause that was tilted and propagating downtail (see Fig. 11 of Kim et al. (2004) as an illustration of the situation). The tilt is probably correlated with the orientation of the shock, but unfortunately for this event we lacked upstream solar wind observations.

\section{Discussion and summary}

We have investigated 10 tail lobe sudden impulse events observed by Cluster during the July-November periods in 2001 and 2002. This is the first statistical study of tail lobe SIs using multispacecraft observations in the lobe, combined with the good knowledge of upstream solar wind conditions. All events in this study were related to strong interplanetary shocks or substantial sudden increases in solar wind dynamic pressure.

Magnetic field signatures of the tail lobe SIs are well described by assuming that a solar wind pressure enhancement squeezes the tail axisymmetrically while moving antisunward. The magnetic field increase is primarily due to increasing (north lobe) or decreasing (south lobe) $B_{x}$ while $B_{y}$ and/or $B_{z}$ exhibit smaller deflections. The amplitudes of the $B_{y}$ deflections were generally larger than the amplitudes of the $B_{z}$ deflections, indicating that the compression of the magnetotail is not symmetric. Perhaps this is because of the differences in the pressure balance between the tail lobe and the solar wind in $Y$ and $Z$ directions. During the SI events the magnetic field vector rotates smoothly in one plane (the plane of maximum variance). The maximum variance direction tends to be parallel to the magnetotail axis which may be deflected from the aberrated GSM $X$-direction by the solar wind flow. The durations of the observed tail lobe SIs varied from 3 to $10 \mathrm{~min}$. This is consistent with the durations of 43 tail lobe SIs reported in Kawano et al. (1992) in the IMP 8 observations (i.e. 3 to $26 \mathrm{~min}$ with the median of $7 \mathrm{~min}$ ). The magnitudes of the Cluster tail lobe SIs ranged between 5.5$27.5 \mathrm{nT}$ which for all expect one event represents $\sim 45-90 \%$ of the initial field magnitude. The SI magnitude is roughly correlated with the increase in solar wind dynamic pressure across the shock. For the observed events the change in the magnetopause shape was not self-similar. Rather, the tail flaring decreased during the compression.

The 2-D Cartesian model by Collier et al. (1998) underestimates the increase in the magnetic field magnitude. When the Petrinec and Russell (1996) model was used to approximate the decrease in the tail radius, the magnetic field increase was best described by the 3-D compression in the limit of small $\Delta$. The final asymptotic magnetic field magnitude in the Collier et al. (1998) model is $(1+\Delta) B_{0}$ while the 3$\mathrm{D}$ compression for the small $\Delta$ gives $(1+2 \Delta) B_{0}$. However when standard least-squares fitting is performed to the data, these two cases yield the same profile. We performed the fitting in the minimum variance coordinate system to take into account the possible magnetotail deflections, as well as the position of Cluster relative to the tail axis. The model describes the behavior of the maximum variance component very well.

The propagation speeds of the SI disturbances obtained using the time delays and the time lagged cross-correlation between the $B_{x}$ profiles between two Cluster spacecraft were 


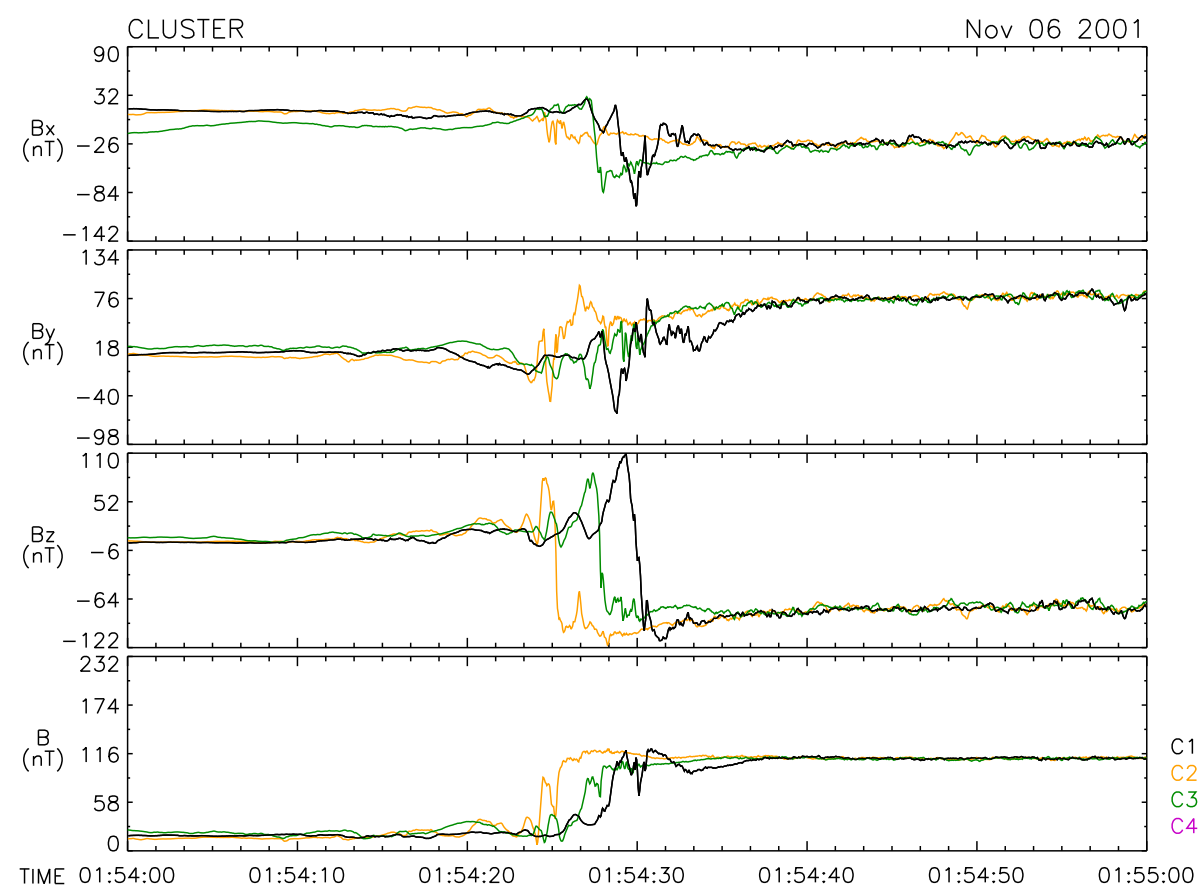

Fig. 17. High resolution Cluster magnetic field measurements on 6 November 2001. Note that a plot covers only a one-minute time interval (01:54:00-01:55:00 UT).

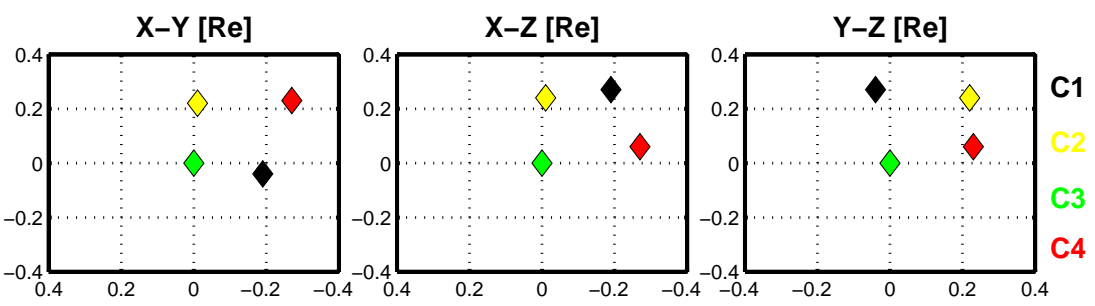

C3 position:

$(-9.45,14.93,2.37)$

Fig. 18. Locations of Cluster spacecraft at 01:53 UT on 6 November 2001 from the reference spacecraft C3 plotted in XY, XZ and YZ planes.

of the order of the solar wind speed rather than the Alfvén speed in the tail lobes $\left(>1000 \mathrm{~km} / \mathrm{s}^{-1}\right)$. Also, the differences between the calculated and observed times between the solar wind shock at the location of WIND/Geotail and the midpoint time of the SI transition at Cluster were small. Thus, it seems that the SI disturbance propagates at a much slower speed than a MHD wave in the tail lobe.

During two of the SI events Cluster became immersed in the magnetospheric boundary layer plasma. The short-time $(\sim 5 \mathrm{~min})$ perturbations in $V_{y}$ and $V_{z}$ indicated the inward movement of the magnetopause. For one event (6 November 2001) Cluster observed a magnetopause crossing. Unfortunately, for this event solar wind plasma data were not available. These events illustrate that the magnetopause is indeed compressed inward at the time that the solar wind pressure enhancement arrives at the location of the spacecraft.
The results of this study are consistent with the tail compression model suggested by Kawano et al. (1992) and by Collier et al. (1998), where the tail lobe SIs are due to the increase in solar wind pressure outside the tail boundary. The magnetotail is assumed to maintain a constant equilibrium while the pressure discontinuity moves downtail. Alternatively, Sugiura et al. (1968) have suggested that tail lobe SIs are due to the magnetic flux carried from the dayside to the tail by a MHD wave resulting from a sudden solar wind pressure increase on the front of the magnetosphere. This idea was based on the time lag between the multispacecraft observations of the solar wind shock and the start of the SI event in the tail lobe on 8 July 1966. However, as discussed here and by Collier et al. (1998), the midpoint time of the SI should be used instead of the start time because of the bending of the field lines ahead of the compression. The magnetopause moves inward behind the pressure discontinuity, 
launching a compressive wave that propagates faster in the magnetosphere than the pressure discontinuity in the magnetosheath (Fairfield, 2003). As a result, the magnetopause expands outward that is observed by small perturbations in the components of velocity and magnetic field before the start of the main SI event (Kim et al., 2004).

The first sign of the SI in the tail lobes may be transmitted as MHD waves, but the results of this study strongly suggest that the dominant cause of the magnetic field changes during tail lobe SIs is the lateral solar wind pressure enhancement. Tail lobe SIs are important because they cause sudden and substantial increases in the tail lobe magnetic field. Although not studied here, the increase in the lobe field must also compress the plasma sheet and may also affect dynamics in that region. Large tail lobe SI events can occur during both southward and northward IMF, but under southward IMF conditions, they are particularly likely to influence tail dynamics. For example, to explain the widening of the auroral oval and the decrease in the polar cap size during solar wind pressure pulses, Boudouridis et al. (2003) suggested that the compression of the magnetotail can significantly increase nightside reconnection. The response of the near-Earth plasma sheet during tail lobe SIs will be the subject of future work.

Acknowledgements. We want to acknowledge C. Mouikis who complied Cluster CIS plots and C. Liebrecht for computational assistance. We thank R. Lepping for the WIND magnetic field data, and A. Lazarus for the WIND solar wind data. We also thank C. W. Smith for the ACE magnetic field data, and D. J. McComas for the ACE solar wind data. These data were obtained through Coordinated Data Analysis Web (CDAWeb). GEOTAIL magnetic field data were provided by S. Kokubun through DARTS at the Institute of Space and Astronautical Science (ISAS) in Japan. The study was supported through the Antares programme of the Academy of Finland.

The editor in chief thanks S. W. H. Cowley and another referee for their help in evaluating this paper.

\section{References}

Araki, T.: Global structure of geomagnetic sudden commencements, Planet. Space Sci., 25, 373-384, 1977.

Araki, T.: A physical model of the geomagnetic sudden commencement, in Solar Wind Sources of Magnetospheric UltraLow-Frequency Waves, Geophys. Monogr. Ser., 81, edited by Engebretson, M. J., Takahashi, K., and Scholer, M., AGU Washington D.C., 183-200, 1994.

Boudouridis, A., Zesta, E., Lyons, L. R., Anderson, P. C., and Lummerzheim, D.: Effect of solar wind pressure pulses on the size and strength of the auroral oval, J. Geophys. Res., 108(A4), 8012, doi:10.1029/2002JA009373, 2003.

Collier, M. R., Slavin, J. A., Lepping, R. P., Ogilivie, K., Laakso, H. and Taguchi, S.: Multispacecraft observation of sudden impulses in the magnetotail caused by solar wind pressure discontinuities: Wind and IMP 8, J. Geophys. Res., 103, 17 293-17 305, 1998.

Echer, E., Gonzalez, W. D., Vieira, L. E., Dal Lago, A., Guarnieri, F. L., Prestes, A., Gonzalez, A. L. C., and Schuch, N. J.: Interplanetary shock parameters during Solar activity maximum (2000) and minimum (1995-1996), Brazilian Journal of Physics, 30, 115122, 2003.

Fairfield, D. H.: Motion of the dusk flank boundary layer caused by solar wind pressure changes and the Kelvin-Helmholtz instability: 10-11 January 1997, J. Geophys. Res., 108,(A12), 1460, doi:10.1029/2003JA010134, 2003.

Kawano, H., Yamamoto, T., Kokubun, S., and Lepping, R.: Rotational polarities of sudden impulses in the magnetotail lobe, J. Geophys. Res., 97, 17 177-17 182, 1992.

Kim, K.-H., Catell, C. A., Lee, D.-H., Balogh, A., Lucek, E., Andre, M., Khotyaintsev, Y., and Rème, H.: Cluster observations in the magnetotail during sudden and quasiperiodic solar wind variations, J. Geophys. Res., 109, A04219, 1-10, doi:10.1029/2003JA010328, 2004.

Lee, D. H. and Hudson, M. D.: Numerical studies on the propagation of sudden impulses in the dipole magnetosphere, J. Geophys. Res., 106, 8435-8445, 2001.

Meurant M., Gerard, J. -C., Hubert, B., Coumans, V., Blockx, C., Ostgaard, N., and Mende, S. B.: Dynamics of global scale electron and proton precipitation induced by a solar wind pressure pulse, G. Res. Lett., 30, 20, 2032, doi:10.1029/2003GL018017, 2003.

Nakai, H., Kamide, Y., and Russel, C. T.: Influences if solar wind parameters and geomagnetic activity on the tail lobe magnetic field: A statistical study, J. Geophys. Res., 96, 5511-5523, 1991.

Petrinec, S. M. and Russell, C. T.: The near-Earth magnetotail shape and size as determined from the magnetopause flaring angle, J. Geophys. Res., 101, 137-52, 1996.

Shue, J. -H., Song, P., Russel, C. T., Gosling, J. T., Steinberg, J. T., Chao, J. K., Zastenker, G., Vaisberg, O. L., Kokubun, S., Singer, H. J., Detman, T. R., and Kawano H.: Magnetopause location under extreme solar wind conditions, J. Geophys. Res., 103, 17 691-17 700, 1998.

Slavin, J. A., Tsurutani, B. T., Smith, E. J., Jones, D. E., and Sibeck, D. G.: Average Configuration of the Distant Magnetotail: Initial ISEE 3 Magnetic Field Results, Geophys. Res. Lett., 10, 973976, 1983.

Smith, E. J., Slavin, J. A., Zwickl, R. D., and Bame, S. J., Shocks and storm sudden commencements, in Solar windmagnetosphere coupling, edited by Y. Kamide and J. A. Slavin, 345-365, Terra Scientific Publishing Company, Tokyo, 1986.

Sonnerup, B. U. O.: Cahill, L. J., Magnetopause structure and attitude from Explorer 12 observation, J. Geophys. Res., 72, 171183, 1967.

Spreiter, J. R., Summers, A. L., and Alksne, A. Y.: Hydromagnetic flow around the magnetosphere, Planet. Space Sci., $14223-$ $14253,1966$.

Sugiura M., Skillman, T. L., Ledley, B. G., and Heppner, J. P.: Propagation of the sudden commencement of July 8, 1966 to the magnetotail, J. Geophys. Res., 73, 6693-6709, 1968.

Szabo A.: An improved solution to the "Rankine-Hugoniot" problem, J. Geophys. Res., 99, 14 737-14 746, 1994.

Zesta, E., Singer, H. J., Lummerzheim, D., Russel, C. T., Lyons, L. R., and Brittnacher, M. J.: The effect of the 10 January 1997, pressure pulse on the magnetosphere-ionosphere current system, in magnetospheric current systems, Geophys. Monogr. Ser., 118, edited by Ohtani, S., Fujii, R., Hesse, M., and Lysak, R. L., AGU, Washington, D.C., 217-226, 2000.

Zhou, X. and Tsurutani, B. T.: Interplanetary shock triggering of nightside geomagnetic activity: Substorms, pseudobreakups, and quiescent events, J. Geophys. Res., 106, 18 957-18 967, 1999. 